\title{
HAWAII QUASAR AND T DWARF SURVEY. I. METHOD AND DISCOVERY OF FAINT FIELD ULTRACOOL DWARFS*,†
}

\author{
Yuko Kakazu ${ }^{1,2}$, Esther M. Hu ${ }^{3}$, Michael C. LiU ${ }^{3}$, Wei-Hao Wang ${ }^{4}$, Richard J. Wainscoat ${ }^{3}$, and Peter L. Capak ${ }^{1}$ \\ ${ }^{1}$ Department of Astronomy, California Institute of Technology, Pasadena, CA 91125, USA; kakazu@ astro.caltech.edu \\ ${ }^{2}$ Insitut d'Astrophysique de Paris, 98 bis boulevard Arago, 75014 Paris, France \\ ${ }^{3}$ Institute for Astronomy, University of Hawaii, 2680 Woodlawn Drive, Honolulu, HI 96822, USA \\ ${ }^{4}$ Academia Sinica Institute of Astronomy and Astrophysics, P.O. Box 23-141, Taipei 10617, Taiwan \\ Received 2009 August 7; accepted 2010 March 3; published 2010 October 8
}

\begin{abstract}
The Hawaii Quasar and T dwarf survey (HQT Survey) is a wide-field, red optical survey carried out with the Suprime-Cam mosaic CCD camera on the $8.2 \mathrm{~m}$ Subaru telescope. The HQT survey is designed to search for low-luminosity $\left(M_{\mathrm{AB} 1450}<-23\right)$ quasars at high redshift $(z>5.7)$ as well as T dwarfs, both of which are selected by their very red $I-z^{\prime}$ colors. We use an optical narrowband filter $N B 816$ to break a well-known $I-z^{\prime}$ color degeneracy between high- $z$ quasars and foreground $\mathrm{M}$ and $\mathrm{L}$ dwarfs, which are more numerous than quasars. This paper is the first in a series of papers from the HQT survey and we report on the discovery of six faint $(19 \leqslant J \leqslant 20)$ ultracool dwarfs found over a $\sim 9.3 \mathrm{deg}^{2}$ area with a limiting magnitude of $z_{\mathrm{AB}}^{\prime} \leqslant 23.3$. These dwarfs were confirmed by near-IR imaging and/or spectroscopy conducted at various facilities on Mauna Kea. With estimated distances of 60-170 pc, these are among the most distant spectroscopically confirmed field brown dwarfs to date. Limits on the proper motions of these ultracool dwarfs suggest that they are old members of the Galactic disk, though future follow-up observations are necessary to minimize errors. Our finding rate of ultracool dwarfs is within model predictions of Liu et al. However, the large brightening amplitude ( $\sim 1 \mathrm{mag})$ previously reported for the $\mathrm{L} / \mathrm{T}$ transition objects appears to overpredict the numbers. We also examine how the survey field latitude affects the survey sensitivity to the vertical scale height of ultracool dwarfs.
\end{abstract}

Key words: brown dwarfs - early universe - quasars: general - stars: low-mass - techniques: photometric

Online-only material: color figures

\section{INTRODUCTION}

Brown dwarfs are substellar objects with masses too low to sustain hydrogen-burning nuclear fusion in their cores. They fill the mass and temperature gap between stars and planets. Studies of these ultracool, low-mass objects therefore provide us with insight into the formation of starlike objects as well as planets.

The last several years have seen tremendous progress in identifications of these ultracool dwarfs thanks to the advent of wide-field surveys carried out on 1-2 m class telescopes: the Sloan Digital Sky Survey (SDSS; York et al. 2000), the Two Micron All Sky Survey (2MASS; Skrutskie et al. 1997), and DENIS (Epchtein et al. 1999). To date, over 100 brown dwarfs with spectral type $\mathrm{T}$, which are the coolest known substellar objects with $T_{\text {eff }} \lesssim 1400 \mathrm{~K}$ (Golimowski et al. 2004; Vrba et al. 2004), have been successfully discovered by these surveys (Chiu et al. 2006, and references therein). However, the shallow depths of these surveys mean that they can only probe brown dwarfs in the solar neighborhood. For example, the shallow SDSS survey can only probe $\mathrm{T}$ dwarfs within $40 \mathrm{pc}$.

Discovery of more distant brown dwarfs allows us to measure their number density and distribution in the Galaxy. The recent advent of large-format near-IR cameras on $4 \mathrm{~m}$ class telescopes has made it possible to identify such a popula-

\footnotetext{
* Based in part on data obtained at the Subaru Telescope, which is operated by the National Astronomical Observatory of Japan.

$\dagger$ Based in part on data obtained at the W. M. Keck Observatory, which is operated as a scientific partnership among the California Institute of

Technology, the University of California, and NASA and was made possible by the generous financial support of the W. M. Keck Foundation.
}

tion: about $80 \mathrm{~T}$ dwarfs within $100 \mathrm{pc}$ have been found in the Large Sky Survey of the United Kingdom Infrared Telescope (UKIRT) Infrared Deep Sky Survey (UKIDSS) (Pinfield et al. 2008; Burningham et al. 2010). The Canada-France Brown Dwarf Survey-InfraRed, carried out with the Wide-field InfraRed Camera (Puget et al. 2004) on the $3.6 \mathrm{~m}$ Canada-FranceHawaii telescope is expected to probe T dwarfs out to $145 \mathrm{pc}$, and a few candidate dwarfs later than $\mathrm{T} 8$ have been reported (Delorme et al. 2010).

These ultracool dwarfs emit $\sim 90 \%$ of their energy at wavelengths longward of $1 \mu \mathrm{m}$ with a peak around $1.2 \mu \mathrm{m}$, and thus near-IR photometry and spectra are advantageous for identifying and studying them. However, a substellar object survey conducted only at near-IR wavelengths is insensitive to late-type $\mathrm{L}$ and early-type $\mathrm{T}$ dwarfs, as the strong $\mathrm{CH}_{4}$ absorption in $1.6 \mu \mathrm{m}$ ( $H$ band) and $2.2 \mu \mathrm{m}$ ( $K$ band) makes these objects look similar to main-sequence stars and asteroids in near-IR colors. On the other hand, optical surveys with far-red bandpasses can search for $\mathrm{T}$ dwarfs over a wide range of temperatures. Moreover, the large field of view (FOV) of optical mosaic CCD cameras with good response at far-red wavelengths enables efficient searches for these ultracool dwarfs, as the discoveries of a number of $\mathrm{T}$ dwarfs in optical surveys demonstrate (e.g., SDSS survey; Chiu et al. 2006, IfA deep survey; Liu et al. 2002).

Intriguingly, quasars at high-redshift $(z \geqslant 5.8)$ exhibit similarly red $I-z^{\prime}$ colors to $\mathrm{L}$ and $\mathrm{T}$ dwarfs due to strong absorption caused by the intervening intergalactic medium (IGM). Indeed, many low-mass stars and brown dwarfs have been found by high $-z$ quasar surveys where these dwarfs were considered contaminants (e.g., Fan et al. 2001; Sharp et al. 2004; 
Willott et al. 2005). Cool dwarfs, particularly $\mathrm{M}$ and L types, are much more common than high- $z$ quasars at the typical depths probed by these surveys. For example, in the shallow SDSS survey, the number density of $\mathrm{M} / \mathrm{L}$ dwarfs is more than 30 times larger than that of $z>5.7$ quasars (Fan et al. 2003). $\mathrm{T}$ dwarfs are rarer than $\mathrm{M} / \mathrm{L}$ dwarfs, but still five times more numerous than quasars. This demonstrates the rarity of quasars and the large numbers of cool dwarfs, particularly $\mathrm{M}$ and $\mathrm{L}$ type dwarfs that are identified by optical color selection.

In order to break the optical color degeneracy between quasars and ultracool dwarfs, near-IR follow-up photometry and/or direct spectroscopy has been used. Despite the recent advent of large near-IR mosaic cameras, however, it is still not very efficient to conduct wide-field near-IR imaging to the required depths. Hence in this paper, we introduce an alternative method to separate quasars from ultracool dwarfs without relying on near-IR data. Our color technique uses an optical narrowband filter $N B 816$ (FWHM $\sim 120 \AA, \lambda_{\text {cen }} \sim 8150 \AA$ ) mounted on the Suprime-Cam mosaic CCD camera on the $8.2 \mathrm{~m}$ Subaru telescope.

Equipped with the new color selection technique, we conducted a wide-field, deep optical survey for high- $z$ quasars $(z>5.7)$ and $\mathrm{T}$ dwarfs using the Suprime-Cam on the Subaru telescope. Hereafter, we refer to this as the Hawaii Quasar and T dwarf survey (HQT). The HQT survey covers an area of about $10 \mathrm{deg}^{2}$ to a limiting magnitude of $z^{\prime} \sim 24$. Our survey is about 4 magnitudes deeper than the SDSS northern Galactic cap survey, which now covers $7500 \mathrm{deg}^{2}$ area (Fan et al. 2006). Our goals are (1) to identify low-luminosity quasars $\left(M_{1450}<\right.$ $-22.5)$ at high $z(z>5.7)$, which are considered to form the bulk of quasar population at high-redshift, and (2) to identify faint, field $\mathrm{T}$ dwarfs and understand their number density and distribution in the Galaxy.

This paper is the first of a series of papers from the HQT survey, in which we describe a survey color selection procedure and report the discovery of several faint $(J>19)$ ultracool dwarfs. The outline of this paper is as follows. In Section 2, we show our survey color selection technique. Survey observations with the Suprime-Cam and follow-up observations using various IR instruments on Mauna Kea are described in Section 3. In Section 4.1, we present classification of our candidates based on their optical and near-IR colors. Near-IR spectra were obtained for three candidates. In Section 4.2, we show that these are indeed ultracool dwarfs and estimate their spectral types from the spectra. Distances and proper motions of the newly identified $\mathrm{T}$ dwarfs are measured and described in Sections 4.3 and 4.4. In Section 4.5 , we calculate the expected number of dwarfs using a simple analytic model and examine their Galactic vertical scale height. We also investigate the effect of field latitude on the scale height for future surveys. Finally, we summarize our results in Section 5. Optical magnitudes in this paper are given in the AB system (Oke 1990), while near-IR magnitudes are in the Mauna Kea Observatory near-IR system (Tokunaga \& Vacca 2005; Leggett et al. 2006) which is a Vega-based photometry system.

\section{COLOR SELECTION TECHNIQUE FOR HIGH $-z$ QSOs $(z>5.7)$ AND T DWARFS}

At $z>5.7$, the $\operatorname{Ly} \alpha$ emission line of quasars begins to move out of the $I$ band and the strong IGM H I absorption below Ly $\alpha$ emission suppresses quasar $I$-band fluxes, making them very red in $I-z^{\prime}$. Thus $z>5.7$ quasars are selected

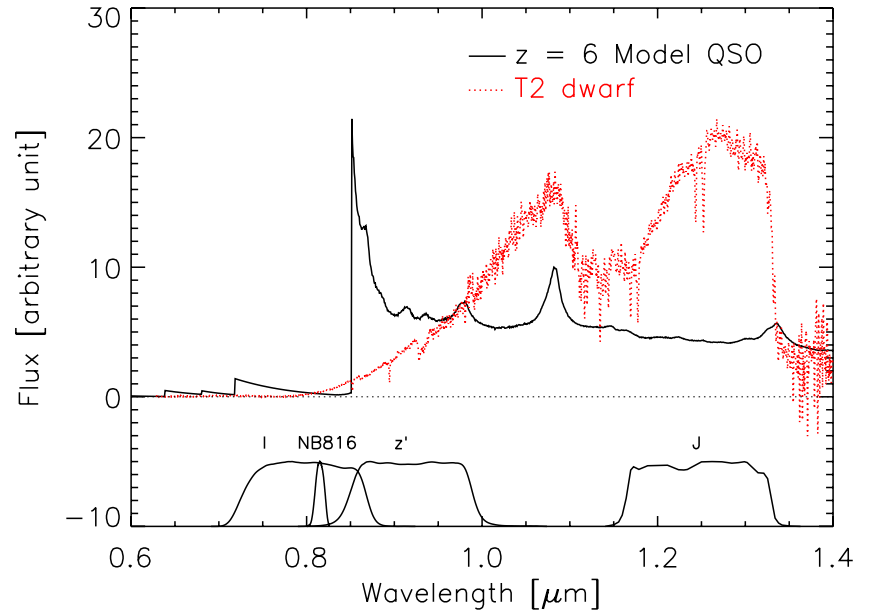

Figure 1. Model quasar spectrum at $z=6$ (black solid line) and IRTF/Spex and Keck/LRIS spectrum of T2 dwarf SDSS1254-0122 from Burgasser et al. (2003) and Cushing et al. (2005)(red dotted line). The transmission profiles of Subaru/Suprime-Cam optical filters $\left(I, N B 816\right.$, and $\left.z^{\prime}\right)$ and the UKIRT/UFTI $J$ band filter are plotted below the spectra as a reference. The strong Ly $\alpha$ emission line of quasars begins to move out of the $I$ band at this redshift. Ultracool dwarfs show a strong infrared excess in comparison to $z=6$ quasars and therefore the $z-J$ color has been used in current high- $z$ quasar surveys to separate quasars from cool dwarfs.

(A color version of this figure is available in the online journal.)

as I-dropouts. Ultracool dwarfs of spectral types M, L, and $\mathrm{T}$ are more numerous than high- $z$ quasars. These cool dwarfs possess similar red $I-z^{\prime}$ colors due to their cool atmospheres, mimicking high- $z$ quasars in optical color selection. Figure 1 illustrates the spectral features of a high- $z$ quasar and an ultracool dwarf.

In order to separate quasars from these foreground cool dwarfs, $z^{\prime}-J$ colors have traditionally been used in optical quasar surveys (e.g., SDSS; Fan et al. 2001), since quasars are bluer due to a declining power-law continuum above Ly $\alpha$, whereas cool dwarfs are red. However, it requires intense telescope time to carry out follow-up $J$-band imaging for all candidates selected by the $I$-dropout technique. For example, our survey with a limiting depth of $z^{\prime} \sim 24$ requires $J$-band imaging data as deep as $J \sim 22$ in order to detect M and L dwarfs, and remove them from the quasar candidates. This means very intensive near-IR observations: it takes $>100 \mathrm{hr}$ even with the Wide Field Camera (WFCAM) on UKIRT, one of the largest IR cameras to image the whole survey area $\left(\sim 10 \mathrm{deg}^{2}\right)$.

We therefore use an optical narrowband filter NB816 as a way of breaking the color degeneracy between quasars and stars without relying on near-IR data. This filter was originally designed to capture $\operatorname{Ly} \alpha$ emission from high- $z$ galaxies (e.g., $\mathrm{Hu}$ et al. 2004; Ajiki et al. 2003), and has also proved to be powerful in identifying extremely metal poor galaxies at $z<1$ (Kakazu et al. 2007; Hu et al. 2009). However, as we show here, it is also powerful for the clean selection of high- $z$ quasars and T dwarfs in I-band drop-out surveys. The NB816 imaging with the Suprime-Cam on Subaru can reach the required depth in ten times less observing time when compared with the $J$ band imaging with UKIRT/WFCAM. Another advantage of the $N B 816$ filter is that it is almost centrally located in the Cousins $I$ filter, thus we can calibrate the $N B 816$ data using Cousins $I$ data. The NB816 filter has a width of $\sim 120 \AA$ (FWHM) and is centered at a nominal wavelength of $8150 \AA$ in a region of low sky background between $\mathrm{OH}$ bands. The nominal specifications 


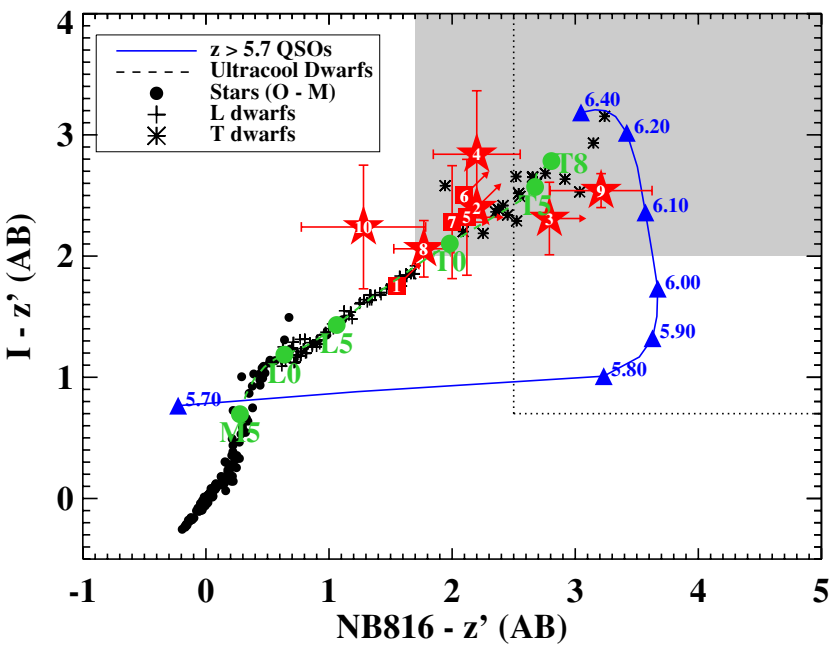

Figure 2. $N B 816-z^{\prime}$ vs. $I-z^{\prime}$ color-color diagram. Foreground objects are shown as asterisks ( $\mathrm{T}$ dwarfs), crosses (L dwarfs), and circles (main-sequence stars). The solid blue line is the track of simulated quasar colors as a function of redshift. The green dashed line is a track of synthesized colors of ultracool dwarfs (M5-T8). Shaded area represents our color selection used in this paper to search for very high $z(z>6.0)$ quasars and T dwarfs. The area surrounded by dotted lines indicates $z>5.7$ quasar and late T dwarf (later than T5) selection. Our candidate quasars and $\mathrm{T}$ dwarfs are shown as red symbols where star symbols indicate that confirmed late-type $\mathrm{L}$ and $\mathrm{T}$ dwarfs and squares are other types of objects. Objects that were detected in only the $z^{\prime}$ band (i.e., HQT-1, HQT-2, HQT-6) are indicated with arrows. Annotated numbers correspond to the labeling in Table 3.

(A color version of this figure is available in the online journal.)

for the Subaru filters may be found at the instrument Web site ${ }^{5}$ and are also described in Ajiki et al. (2003).

Figure 2 presents the $N B 816-z^{\prime}$ versus $I-z^{\prime}$ color-color diagram for known stars and brown dwarfs with the predicted quasar track as a function of redshift overplotted. The quasar color (blue solid line) was computed from the SDSS composite quasar spectrum of Vanden Berk et al. (2001) at redshifts 5.7-6.4. We first extrapolated the spectrum with a $f_{v} \sim v^{-0.46}$ power-law continuum below Ly $\alpha$, and then applied the Songaila \& Cowie (2002) IGM transmission law for a given redshift of quasar spectrum. Finally, we convolved results through the Suprime-Cam filter response profiles, which include the CCD quantum efficiency, atmospheric absorption, and optics effects. To compute stellar colors, we convolved the stellar spectral flux library of Pickles (1998) with the Suprime-Cam filter response profile. For ultracool stars, we used Sandy Leggett's online database $^{6}$ and IRTF/SpeX spectra from Cushing et al. (2005). A seventh-order polynomial fit to the synthesized colors was computed as a function of spectral type of ultracool dwarfs and is shown as the pink dashed track in Figure 2. Table 1 lists the coefficients of the polynomial fits to optical and near-IR colors of dwarfs of M-T types. The optical-near-IR colors $\left(z^{\prime}-J\right)$ and pure near-IR colors of dwarfs were computed in the same manner as optical colors, but for near-IR bandpasses we used the Mauna Kea consortium filter set installed in UKIRT/UFTI. The fits to pure near-IR colors $(J-H$ and $J-K)$ are made from both synthesized and observational data from Knapp et al. (2004).

As can be seen in this diagram, $z>5.7$ quasars are selected as $N B 816$ dropouts (i.e., have red $N B 816-z^{\prime}$ colors). On the

\footnotetext{
5 http://www.naoj.org/Observing/Instruments/SCam/sensitivity.html

6 http://www.jach.hawaii.edu/UKIRT/
}

other hand, $\mathrm{M}$ and $\mathrm{L}$ dwarfs, which are the dominant population of $I$-dropouts at the typical depths probed by quasar surveys (e.g., Fan et al. 2001), have bluer NB816 - $z^{\prime}$ colors. Hence, we can separate quasars from $\mathrm{M}$ and $\mathrm{L}$ dwarfs by using their $N B 816-z^{\prime}$ colors. The reason why high- $z$ quasars are selected as NB816-dropouts is easily understood. At $z \sim 5.7$, Ly $\alpha$ emission from the quasar comes into the NB816 filter and at $z \sim 5.8$ it moves out of the NB816 filter. This quasar redshift transition at $z=5.7 \rightarrow 5.8$ is marked by a huge increase in the $N B 816-z$ color term. At $z>5.8$, the strong Ly $\alpha$ forest absorption depresses the flux in the $N B 816$ band, leaving very little flux from the quasar in this band. This effect shows up as nearly constant $N B 816-z^{\prime}$ colors. At $z \sim 6.1$, Ly $\alpha$ flux comes into the $z^{\prime}$ filter and the forest absorption now starts to affect the $z^{\prime}$-band flux as well. This makes NB816 - $z^{\prime}$ slightly bluer at the highest redshift range $(z>6.1)$.

In the extremely red regime (e.g., $I-z^{\prime}>2.0$ ), however, $N B 816-z^{\prime}$ colors of quasars and T dwarfs become degenerate. This is because both quasars and T dwarfs have very little flux in the NB816 and I-band filters; the former due to strong IGM absorption and the latter due to strong $\mathrm{K} I$ absorption. Thus, nearIR follow-ups or direct spectroscopy are required to disentangle these two populations.

However, since the majority of $I$-dropouts in the high- $z$ quasar surveys are Galactic $\mathrm{M}$ and $\mathrm{L}$ dwarfs (i.e., T dwarfs and quasars are much rarer), our technique is useful to remove such populations from the candidates. In our survey, we are interested in both high- $z$ quasars and T dwarfs - both of which are of great scientific interest in their own right. The color criteria for $z>5.7$ quasars and late T dwarfs (later than T5) are shown as a region surrounded by dotted lines in Figure 2, and it is expressed as

$$
I-z^{\prime}>0.7
$$

and

$$
N B 816-z^{\prime}>1.7
$$

where magnitudes are in the $\mathrm{AB}$ system.

In this paper, we discuss extremely red objects with $I-z^{\prime}>$ 2.0 colors found in the HQT survey, with the aim of finding very high $z$ quasars $(z>6)$ and T dwarfs (T0-T9). This selection is shown as the shaded area in Figure 2. The high$z(5.7<z<6.0)$ quasar candidates with moderately red $I-z^{\prime}$ colors $\left(0.7<I-z^{\prime}<2.0\right)$ found by the HQT survey will be discussed in future papers.

In the HQT survey, instead of using the Sloan $i^{\prime}$ filter, we chose to use the Cousins $I$ filter since its effective wavelength matches better with the $N B 816$. The Sloan $i^{\prime}$ filter has a shorter effective wavelength than the Cousins $I$, making $i^{\prime}-z^{\prime}$ of a quasar at the same redshift greater than $I-z^{\prime}$. Nonetheless, in either $I$ or $i^{\prime}$, one can use the $N B 816-z^{\prime}$ color criteria to eliminate $\mathrm{M}$ and $\mathrm{L}$ dwarfs from the candidates.

\section{OBSERVATIONS}

\subsection{Subaru/Suprime-Cam Imaging Survey}

The optical imaging data were obtained in a number of runs between 2002 September and 2006 September with the Suprime-Cam (Miyazaki et al. 2002) on the $8.2 \mathrm{~m}$ Subaru telescope. The Suprime-Cam consists of ten $2048 \times 4096$ CCD chips, yielding an FOV of $34^{\prime} \times 27^{\prime}$ with a pixel scale of 0 .2. Its wide area coverage and good response at red optical wavelengths make this instrument ideal for surveys for high- 
Table 1

Coefficients of Polynomial Fits to Colors of Ultracool Dwarfs

\begin{tabular}{|c|c|c|c|c|c|c|c|c|}
\hline Color & $\mathrm{c} 0$ & $\mathrm{c} 1$ & $\mathrm{c} 2$ & c3 & $\mathrm{c} 4$ & $\mathrm{c} 5$ & c6 & c7 \\
\hline$I-z^{\prime}$ & $1.187 \mathrm{e}+00$ & $3.235 \mathrm{e}-02$ & $-5.306 e-03$ & $2.044 \mathrm{e}-03$ & $-4.500 \mathrm{e}-06$ & $-1.555 \mathrm{e}-05$ & $7.703 e-07$ & $-9.030 \mathrm{e}-09$ \\
\hline$i^{\prime}-z^{\prime}$ & $1.529 \mathrm{e}+00$ & $3.463 \mathrm{e}-02$ & $-2.713 e-03$ & $2.779 \mathrm{e}-03$ & $-6.910 \mathrm{e}-05$ & $-1.951 \mathrm{e}-05$ & $1.292 \mathrm{e}-06$ & $-2.226 \mathrm{e}-08$ \\
\hline$N B 816-z^{\prime}$ & $6.371 \mathrm{e}-01$ & $7.816 \mathrm{e}-02$ & $-2.119 \mathrm{e}-03$ & $4.426 \mathrm{e}-05$ & $1.643 e-04$ & $-2.139 \mathrm{e}-06$ & $-1.132 \mathrm{e}-06$ & $4.324 \mathrm{e}-08$ \\
\hline$z^{\prime}-J$ & $2.613 \mathrm{e}+00$ & $1.705 e-01$ & $-1.728 \mathrm{e}-02$ & $-1.546 \mathrm{e}-03$ & $2.201 \mathrm{e}-04$ & $5.674 \mathrm{e}-06$ & $-1.040 \mathrm{e}-06$ & $2.398 \mathrm{e}-08$ \\
\hline$J-H$ & $6.832 \mathrm{e}-01$ & $4.917 \mathrm{e}-02$ & $2.109 \mathrm{e}-03$ & $-2.465 e-04$ & $-2.299 \mathrm{e}-05$ & $-2.046 \mathrm{e}-06$ & $9.277 \mathrm{e}-08$ & $2.987 \mathrm{e}-09$ \\
\hline$J-K$ & $1.197 \mathrm{e}+00$ & $8.577 \mathrm{e}-02$ & $7.732 \mathrm{e}-03$ & $-1.726 \mathrm{e}-06$ & $-1.980 \mathrm{e}-04$ & $-4.205 e-06$ & $1.260 \mathrm{e}-06$ & $-3.253 \mathrm{e}-08$ \\
\hline
\end{tabular}

Notes. These are the coefficients of the seventh-order polynomial fits for the optical and near-IR colors of M -T dwarfs, and are used in Figure 2 and Figures 8-10. Note that a Cousins $I$ filter is used in the HQT survey, however, we also list Sloan $i^{\prime}-z^{\prime}$ colors as a reference for interested readers. The fits are defined as

$$
\text { Color }=\sum_{i} c_{i} \times(\mathrm{SpT})^{i}
$$

where $\mathrm{SpT}=-10$ for $\mathrm{M} 0, \mathrm{SpT}=0$ for $\mathrm{L} 0, \mathrm{SpT}=10$ for $\mathrm{T} 0$, and $\mathrm{SpT}=19$ for $\mathrm{T} 9$.

Table 2

Survey Area and Ultracool Dwarf Number Counts

\begin{tabular}{lccccc}
\hline \hline Field Name & R.A. Range & Decl. Range & $\begin{array}{c}\text { Galactic Latitude } \\
(\mathrm{deg})\end{array}$ & $\begin{array}{c}\text { Survey Area } \\
\left(\mathrm{deg}^{2}\right)\end{array}$ & $\begin{array}{c}\text { Used Area } \\
\left(\mathrm{deg}^{2}\right)\end{array}$ \\
\hline A2218-wide & $16: 14: 10.40-16: 42: 28.00$ & $+65: 10: 07.30$ to $+66: 50: 09.95$ & 39.0 & 3.50 & 1.87 \\
A370-wide & $02: 36: 11.13-02: 45: 19.71$ & $-03: 03: 04.82$ to $-00: 29: 04.89$ & -53.0 & 5.25 & 3.00 \\
SSA22-wide & $22: 10: 28.21-22: 21: 28.39$ & $-00: 01: 02.96$ to $+02: 05: 57.00$ & -43.0 & 5.25 & 4.42 \\
\hline Total & & & & 14.0 & 9.30 \\
\hline
\end{tabular}

Notes. "Survey Area" means the area observed with the Subaru/Suprime-Cam in three bandpasses $\left(I, N B 816, z^{\prime}\right)$. "Used Area" shows the area used in this paper and satisfies the depths' requirement.

$z$ objects and ultracool dwarfs, both of which are very red at optical wavelengths.

The HQT survey area consists of three contiguous fields surrounding Abell 2218 (A2218-wide; R.A.cen $\approx$ 16:30, decl.cen $\left._{\text {. }} \approx+66: 00\right)$, SSA22 (SSA22-wide; R.A.cen $=22: 15$, decl.cen $_{\text {. }}+01: 00$ ), and Abell 370 (A370-wide; R.A.cen $=$ 02:41, decl.cen $=-01: 44)$. Two survey fields, A2218-wide and A370-wide, include massive galaxy clusters. However, the area affected by these clusters is very small compared to the total survey area. Therefore, little effect from cluster properties such as a lensing magnification is expected. The fields were chosen to be at low extinction and to overlap with known, wellstudied fields for which we already have ultradeep Suprime-Cam imaging data for the central regions $\left(\sim 0.2 \mathrm{deg}^{2}\right)$ from narrowband high- $z$ galaxy studies (Hu et al. 2002, 2004, 2005). The main advantage to choosing such fields is that it permits us to check the wide shallow photometry and also provides us with color calibration information in the event of non-photometric conditions.

Observations with the Suprime-Cam were taken as a series of three-point dithers, with typical exposure times on each Suprime-Cam field of $180 \mathrm{~s}$ in $I$, and $540 \mathrm{~s}$ in NB816 and $z^{\prime}$. Data that were taken under poor weather conditions- that is, extinction greater than $0.5 \mathrm{mag}$ and/or seeing sizes greater than $1^{\prime \prime}$-were re-taken under better weather conditions. In order to achieve uniform survey depths, we only use the central $25^{\prime} \times 25^{\prime}$ area from each Suprime-Cam pointing. Most of our $I, N B 816$, and $z^{\prime}$-band images were obtained nearly simultaneously to avoid transients such as supernovae and Kuiper Belt objects. The data were reduced in a similar manner to that described in Capak et al. (2004) which is based on the Imcat software developed by Nick Kaiser. ${ }^{7}$ Calibrations were done using spectrophotometric standard stars when available. Data that were obtained under

\footnotetext{
7 Imcat is available at http://www.ifa.hawaii.edu/ kaiser/imcat.
}

non-photometric weather conditions were cross-calibrated using stars in the overlapping area between each Suprime-Cam field. For the SSA22-wide and A370-wide fields, existing ultradeep Suprime-Cam data of the central fields were used to cross-check the photometric zero points. The $I-N B 816$ and $I-z^{\prime}$ colors of field stars were also examined to ensure the accuracy of photometric zero points. Photometric measurements were made using SExtractor version 2.2.2 (Bertin \& Arnouts 1996) with the "double image" mode to measure $I$ and NB816 magnitudes for all objects using the $z^{\prime}$-band image as the detection frame (i.e., $I$ and NB816 photometry were made at the positions of sources detected in the $z^{\prime}$ band). Since each Suprime-Cam field was observed under a different seeing condition (but always better than $1^{\prime \prime}$ seeing), we used an aperture diameter of $2.5 \times$ seeing (FWHM) which is between 1". 6 and 2". 4 .

In order to determine the survey area as a function of depths, we first formed a sensitivity map (i.e., rms map) by taking the inverse square root of the exposure map. The sensitivity map was then scaled to have the flux unit using the magnitude zero points and aperture diameter used for each Suprime-Cam field. Next, we made the histogram of the scaled sensitivity map to derive the relation of depths and number of pixels which have the same depths. The survey area was then computed by integrating pixels. Figure 3 shows the resulting survey area versus depth in three filter bandpasses $\left(I, N B 816\right.$, and $\left.z^{\prime}\right)$. This shows that about $\approx 14 \mathrm{deg}^{2}$ area are covered to $5 \sigma$ limiting depths of $I \approx 23.8$, $N B 816 \approx 23.4$, and $z^{\prime} \approx 23.5$, which are indicated by a blue dotted line for each band.

We selected candidates at $z^{\prime} \leqslant 23.3$. This is slightly brighter than the $5 \sigma$ limit of 23.5 because it is the depths at $I$ and NB816 that really limit us. In order to satisfy the color criteria for $z>6.1$ QSO and T dwarfs, our candidates need to be very red (i.e., $I z>2.0$ and $N B 816 z>1.7$ ). This means that we need to probe deeper in $I$ and $N B 816$ with respect to the 

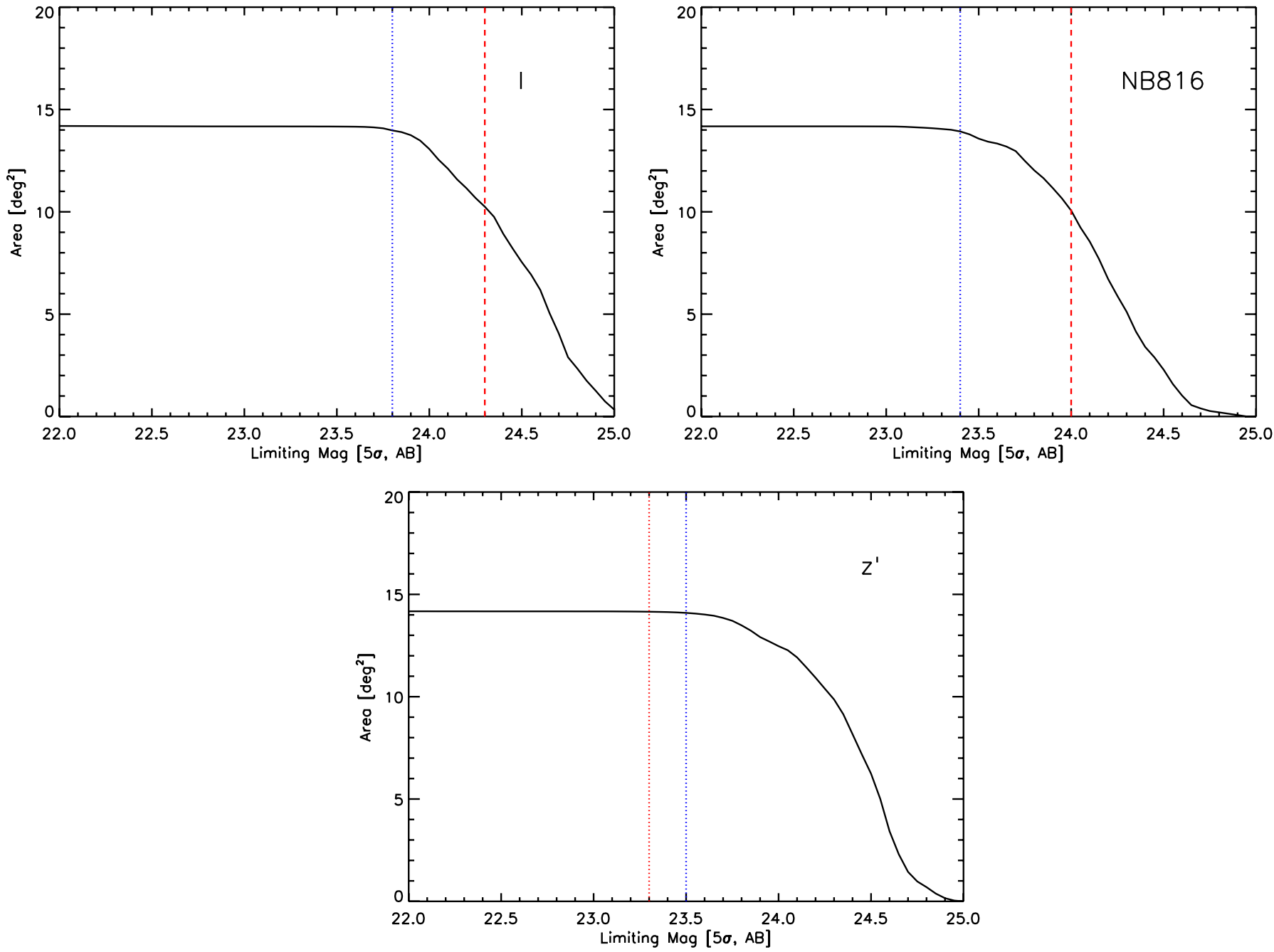

Figure 3. HQT survey area $\left(\mathrm{deg}^{2}\right)$ as a function of $5 \sigma$ limiting magnitude in $I, N B 816$, and $z^{\prime}$ bands. The blue dotted lines indicate $5 \sigma$ limiting magnitudes for about a $14 \mathrm{deg}^{2}$ area, and these are $I=23.8, N B 816=23.4, z^{\prime}=23.5$. The red dashed lines shown in $I$ and $N B 816$ bands are the depths required for the color selection of candidates at $z^{\prime} \leqslant 23.3$. About a $10 \mathrm{deg}^{2}$ area satisfies this condition in individual bandpasses. Of them, we use $9.3 \mathrm{deg}^{2}$ which satisfies the condition in all three bands simultaneously.

(A color version of this figure is available in the online journal.)

$z^{\prime}$ band. We require the above two color selections to be at $\gtrsim 2 \sigma$ confidence levels. We therefore restricted our search to the area where $I$ and NB816 are sufficiently deep $\left(I_{2 \sigma} \geqslant 25.3\right.$ and $N B 816_{2 \sigma} \geqslant 25.0$; corresponding to $5 \sigma$ limiting magnitudes of 24.3 and 24.0, respectively, which are shown as red dashed lines in Figure 3). Our final survey area is $9.3 \mathrm{deg}^{2}$, of which 1.9 $\mathrm{deg}^{2}$ is in A2218-wide, $3.0 \mathrm{deg}^{2}$ in A370-wide, and $4.4 \mathrm{deg}^{2}$ in SSA22-wide. The location of our survey field and area coverage are summarized in Table 2.

\subsection{QSO and T dwarf Candidate Selection}

With the purpose of finding $\mathrm{T}$ dwarfs and quasars at very high $z \quad(z>6.1)$, we selected extremely red objects with $I-z^{\prime}>2.0$ and $z^{\prime}<23.3$. Quasars at the lower redshift range $(5.7<z<6.1)$ with moderately red colors $\left(1.0<I-z^{\prime}<2.0\right)$ will be discussed in a future paper. When applying the $I-z^{\prime}$ and $N B 816-z^{\prime}$ color criteria, we included all objects which satisfy the conditions within $1 \sigma$ errors in colors (i.e., all objects whose colors with $1 \sigma$ uncertainty fall in the shaded area in Figure 2). This was done because photometric errors in $I$ and $N B 816$ are relatively large due to the shallowness of the image in this bandpass.
We examined all selected objects with an eye to removing transients and spurious detections due to spikes from bright stars, cosmic rays, and artifacts. In total, 10 objects (five in A2218-wide, three in A370-wide, and two in SSA22-wide) were chosen as final high- $z$ quasar $(z>6)$ and T dwarf candidates. Their coordinates and photometric properties are summarized in Table 3.

Figure 4 presents optical and near-IR images of one of our candidates, HQT-11, as an example. Our candidates exhibit extremely red $I-z^{\prime}$ and $N B 816-z^{\prime}$ colors (i.e., luminous in $z^{\prime}$ but very faint in $I$ and NB816), suggestive of high- $z$ quasars or T dwarfs. We checked that all our selected candidates in the field surrounding galaxy clusters (A370 and A2218) are $>30^{\prime}$ away from the center of clusters. Since the projected virial radii are less than $12^{\prime}$ for these clusters (e.g., Medezinski et al. 2010; Ko et al. 2009) the lensing effect on our candidates is negligible.

\subsection{Follow-up Near-IR Imaging}

We carried out follow-up near-IR observations using various facilities on Mauna Kea. Table 4 summarizes our near-IR 


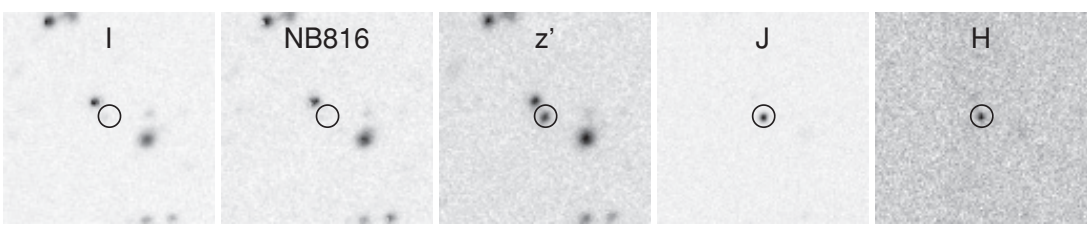

Figure 4. Optical Suprime-Cam and near-IR MOIRCS images of HQT-9 shown with a circle of 1 1.2 radius. Each image is a $20^{\prime \prime}$ side. This object was selected because of its extremely red $I-z^{\prime}$ and $N B 816-z^{\prime}$ colors, suggesting either a high- $z$ quasar or a T dwarf. Follow-up near-IR imaging $(J$ and $H)$ shows a clear detection, indicating a $\mathrm{T}$ dwarf. Further spectroscopy (see Figure 7) confirmed this object to be a T6 dwarf.

Table 3

Photometric Properties of Candidates

\begin{tabular}{|c|c|c|c|c|c|c|c|c|c|c|}
\hline Name & R.A. & Decl. & $z^{\prime}$ & $J$ & $H$ & $I-z^{\prime}$ & $N B 816-z^{\prime}$ & $z^{\prime}-J$ & $J-H$ & $J-K$ \\
\hline HQT-1 & $16: 23: 31.78$ & $+65: 34: 36.39$ & $23.3(0.13)$ & $22.0(0.11)$ & & $>1.75$ & $>1.55$ & $1.28(0.17)$ & & \\
\hline HQT-2* & $16: 28: 25.99$ & $+65: 25: 49.07$ & $23.3(0.07)$ & $20.1(0.09)$ & $19.6(0.07)$ & $>2.40$ & $>2.20$ & $3.15(0.11)$ & $0.57(0.11)$ & $\cdots$ \\
\hline HQT-3* & $16: 34: 13.34$ & $+65: 18: 12.65$ & $22.7(0.04)$ & $19.7(0.07)$ & $18.8(0.04)$ & $2.31(0.30)$ & $>2.79$ & $2.96(0.08)$ & $0.92(0.08)$ & \\
\hline HQT-5 & $16: 18: 52.44$ & $+66: 37: 03.83$ & $22.8(0.07)$ & $20.2(0.02)$ & & $2.32(0.48)$ & $>2.12$ & $2.66(0.08)$ & $\ldots$ & \\
\hline HQT-6 & 02:45:01.67 & $-02: 05: 36.68$ & $23.3(0.07)$ & $>21.3$ & & $>2.50$ & $>2.10$ & $<2.00$ & & \\
\hline HQT-7 & 02:44:52.77 & $-02: 06: 49.42$ & $23.4(0.08)$ & $>21.3$ & & $2.28(0.47)$ & $>2.00$ & $<2.10$ & .. & $\ldots$ \\
\hline HQT-10* & $22: 21: 26.92$ & $+01: 03: 51.12$ & $23.3(0.12)$ & $20.0(0.15)$ & $19.9(0.16)$ & $2.24(0.51)$ & $1.28(0.50)$ & $3.30(0.19)$ & $0.07(0.22)$ & $\ldots$ \\
\hline
\end{tabular}

Notes. Optical magnitudes $\left(I, z^{\prime}, N B 816\right)$ are given in the AB system, while near-IR magnitudes are in the Vega-based system. Objects with asterisks are probable T-type dwarfs from their near-IR colors, though HQT-3 could be a late L dwarf. Two-sigma magnitude and color limits are shown for the objects that were not detected in the $I, N B 816$, or $J$ band.

Table 4

Log of Near-IR Imaging Observations

\begin{tabular}{|c|c|c|c|c|c|}
\hline Name & Filter & UT Date & Instrument & Exp. Time [s] & Weather [seeing] \\
\hline HQT-1 & $J$ & 2007.04 .29 & Subaru/MOIRCS & 720 & Photometric [0,4] \\
\hline \multirow[t]{2}{*}{ HQT-2 } & $J$ & 2005.07.21 & Subaru/CISCO & 660 & Photometric [0,'7] \\
\hline & $H$ & 2005.07.21 & Subaru/CISCO & 660 & Photometric [0,7] \\
\hline \multirow[t]{2}{*}{ HQT-3 } & $J$ & 2005.07.21 & Subaru/CISCO & 660 & Photometric [0,7] \\
\hline & $H$ & 2005.07.21 & Subaru/CISCO & 660 & Photometric $\left[00^{\prime \prime} 7\right]$ \\
\hline \multirow[t]{2}{*}{ HQT-4 } & $J$ & 2005.07.21 & Subaru/CISCO & 660 & Photometric [0,7] \\
\hline & $H$ & 2007.06.12 & Subaru/MOIRCS & 135 & Photometric [0'.5] \\
\hline HQT-5 & $J$ & 2007.04.29 & Subaru/MOIRCS & 720 & Photometric [0,4] \\
\hline HQT-6 & $J$ & 2005.08 .30 & UKIRT/WFCAM & 240 & Thin cirrus $\left[2^{\prime \prime}\right]$ \\
\hline HQT-7 & $J$ & 2005.08 .30 & UKIRT/WFCAM & 240 & Thin cirrus $\left[2^{\prime \prime}\right]$ \\
\hline \multirow[t]{2}{*}{ HQT-8 } & $J$ & 2007.01 .06 & Subaru/MOIRCS & 1080 & Cirrus $\left[1^{\prime \prime}\right]$ \\
\hline & Ks & 2007.01 .06 & Subaru/MOIRCS & 150 & Cirrus $\left[1^{\prime \prime}\right]$ \\
\hline \multirow[t]{2}{*}{ HQT-9 } & $J$ & 2007.06 .12 & Subaru/MOIRCS & 720 & Photometric [0'.5] \\
\hline & $H$ & 2007.07.08 & UKIRT/UIST & 1440 & Photometric $[0,7]$ \\
\hline \multirow[t]{2}{*}{ HQT-10 } & $J$ & 2005.05 .08 & UKIRT/WFCAM & 240 & Photometric $\left[2^{\prime \prime}\right]$ \\
\hline & $H$ & 2007.07.08 & UKIRT/UIST & 1440 & Photometric $\left[0^{\prime \prime} 7\right]$ \\
\hline
\end{tabular}

observations. Photometric measurements were made again with an aperture diameter of $2.5 \times$ seeing $(\mathrm{FWHM})$. In the following subsections, we describe our follow-up observations.

\subsubsection{UKIRT/WFCAM Imaging}

The $J$-band imaging data of two candidates (HQT-6 and HQT-7) were obtained with the near-IR WFCAM (Casali et al. 2007) installed on the $3.8 \mathrm{~m}$ UKIRT. WFCAM is made of four $2048 \times 2048$ arrays, achieving a large FOV of $0.2 \mathrm{deg}^{2}$ with a relatively large pixel scale of 0.4 . The total integration time for each quasar/T dwarf candidate is 4 minutes. The data were pipeline processed by the Cambridge Astronomical Survey Unit, and field 2MASS stars were used to calibrate the data.

Our data were obtained during the initial phase of WFCAM commissioning when there was an issue with image quality and cosmetics (see Dye et al. 2006). As a result, the stellar pointspread function was as large as $2^{\prime \prime}$ and therefore we used a large aperture diameter $\left(\sim 5^{\prime \prime}\right)$ for the photometric measurements of the candidates.

\subsubsection{Subaru/CISCO and MOIRCS Imaging}

All of our candidates except two (HQT-6 and HQT-7) were observed with the near-IR instruments on Subaru; CISCO (Motohara et al. 2002) and MOIRCS (Ichikawa et al. 2006). CISCO is a $1024 \times 1024$ HAWAII array, covering a $1.8 \times 1.8$ area with a pixel scale of $0^{\prime \prime} 11$. MOIRCS is a successor to CISCO and has capabilities for both wide-field imaging $\left(\sim 4^{\prime}\right.$ $\times 7^{\prime}, 0^{\prime} \prime 117$ pixel $\left.^{-1}\right)$ and multi-object spectroscopy. MOIRCS employs two HAWAII-2 $2048 \times 2048$ arrays.

All the data except for those of HQT- 8 were obtained under photometric conditions with better than 0.5 seeing, and were 


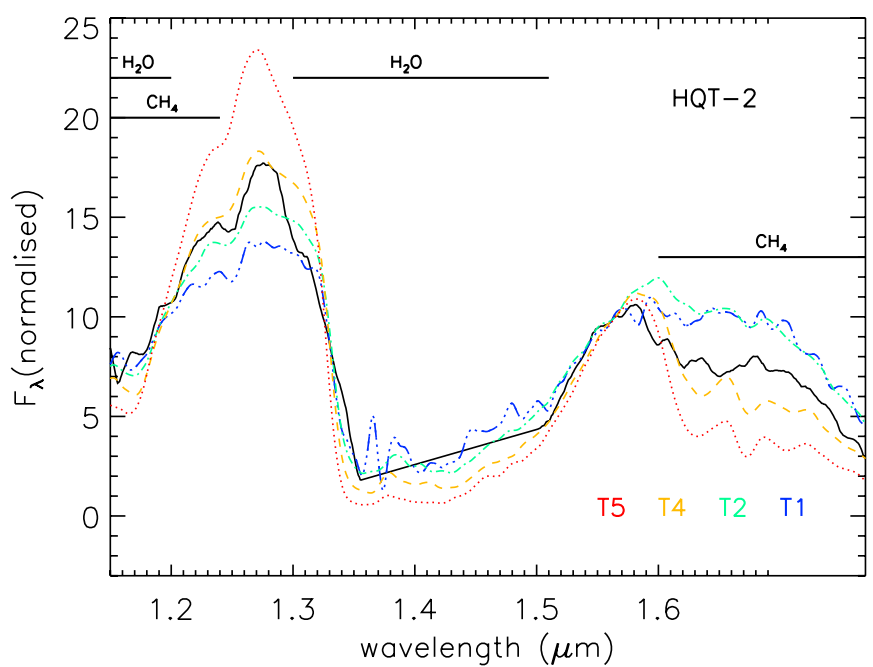

Figure 5. Subaru/CISCO $J H$ spectrum of HQT-2 (black solid curve). Also shown for comparison are the standard spectra for $\mathrm{T}$ dwarfs from Burgasser et al. (2006) (T5 [2MASS J15031961+2525196]: red dotted curve, T4 [SDSS J22541892+3123498]: orange dashed curve, T2 [SDSS J125453.90-012247.4]: green dash-dotted curve, T1 [SDSS J083717.21-000018.0]: blue dash-dot-dot-dotted curve). Major $\mathrm{H}_{2} \mathrm{O}$ and $\mathrm{CH}_{4}$ absorption bands, which are indicative of $\mathrm{T}$ dwarfs, are clearly detected. This object is classified as T2-T4 based on the scheme of Burgasser et al. (2006).

(A color version of this figure is available in the online journal.)

calibrated by using UKIRT faint standard stars FS23 and FS139 in the Mauna Kea near-IR photometric system (Leggett et al. 2006). The HQT-10 data were obtained under cirrus and thus were calibrated by 2MASS stars in the field. Data were processed by the IDL based pipeline SIMPLE developed by W.-H. Wang (Wang et al. 2010) ${ }^{8}$.

\subsubsection{UKIRT/UIST Imaging}

The $H$-band imaging data of HQT-9 and HQT-10 were taken with the UKIRT Imager-Spectrometer (UIST; Ramsay Howat et al. 2004) on the $3.8 \mathrm{~m}$ UKIRT telescope under photometric condition. UIST contains a $1024 \times 1024 \mathrm{InSb}$ array with two plate scales of $0^{\prime} \cdot 12$ and $0^{\prime \prime} 06$, yielding an FOV of $2^{\prime} \times 2^{\prime}$ or $1^{\prime} \times 1^{\prime}$. Data were pipeline-processed by ORAC-DR recipes provided by the UKIRT. ${ }^{9}$

\subsubsection{Subaru/CISCO Spectroscopy of HQT-2 and HQT-3}

The $J H$ grism spectra of HQT-2 and HQT-3 were obtained with Subaru/CISCO on 2005 July 20 UT under conditions of cirrus with a typical seeing FWHM of $1^{\prime \prime}$. A set of 12 and 14 exposures of $300 \mathrm{~s}$ were obtained for HQT-2 and HQT-3, respectively, both in an ABBA dither pattern with a $10^{\prime \prime}$ offset. We used the $1^{\prime \prime}$ slit, yielding a spectral coverage from 1.1 to $1.8 \mu \mathrm{m}$ with a resolving power of $R=\lambda / \Delta \lambda \approx$ 200. The A0V star SAO 030187 was observed immediately after the target exposures at a similar airmass. Data were reduced using custom-made IDL scripts and IRAF/apall for the spectral extraction. First images were pairwise subtracted and divided by a median-combined flat-field image. Images obtained at the same nod position were then median-combined. Extractions of one-dimensional spectra were done with IRAF/ apall. The transformation between pixel and wavelength is fixed

\footnotetext{
8 SIMPLE is available at http://www3.asiaa.sinica.edu.tw/ whwang/ idl/SIMPLE/index.htm.

9 http://www.oracdr.org
}

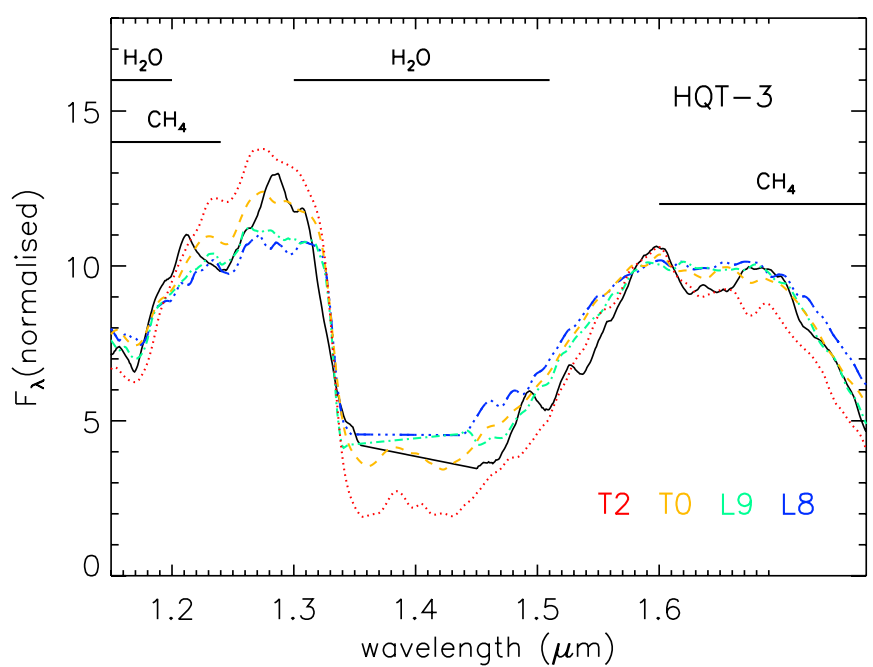

Figure 6. Subaru/CISCO $J H$ spectrum of HQT-3 (black solid curve). The comparison with the overplotted $\mathrm{T}$ dwarf standard of Burgasser et al. (2006) and L dwarf spectra of Geballe et al. 2002 (T2 [SDSS J125453.90-012247.4]: red dotted curve, T0 [SDSS J0423-0414]: orange dashed curve, L9 [SDSS 083008.12+482847.4]: green dash-dotted curve, L8 [2MASS J152322.6+301456]: blue dash-dot-dot-dotted curve) suggests this object to be a late-type $\mathrm{L}$ or an early-type $\mathrm{T}$.

(A color version of this figure is available in the online journal.)

for CISCO and thus wavelength calibration was done by using the relation provided at the CISCO Web site. ${ }^{10}$ Wavelength was independently calibrated using the night sky lines, and this method gave consistent results. Hydrogen absorption features intrinsic to the atmosphere of a A0 V star were removed by linear interpolation. In order to correct for telluric absorption and instrument response, we divided the extracted spectra by the spectrum of an A0 V calibration star and multiplied by a $9480 \mathrm{~K}$ blackbody curve. Finally, the spectra obtained at different nod positions (A and B) were combined. Due to the faintness of the targets $(J \sim 20)$ and the presence of cirrus, the signal-tonoise ratio $(\mathrm{S} / \mathrm{N})$ of the resulting spectra was quite low. We then smoothed the spectra with a boxcar of 50 pixels. Figures 5 and 6 show final spectra for HQT-2 and HQT-3 with a spectral resolution $R=40$.

\subsubsection{Keck/NIRSPEC Spectroscopy of HQT-9}

The $J$-band Spectrum of HQT-9 was obtained on 2004 December 5 UT with the KeckII/NIRSPEC (McLean et al. 1998) under non-photometric, very windy ( $>30$ knots) weather conditions. NIRSPEC employs a $1024 \times 1024 \mathrm{InSb}$ array and has a high (Echelle) resolution mode $(R \approx 25,000)$, as well as a low-resolution mode $(R \approx 2,200)$. A total of $10 \mathrm{~min}$ utes of exposure was obtained in the low-resolution mode using the NIRSPEC-3 blocking filter which approximates the $J$ band. A 0.76 slit width was used. The observation was taken at two different positions along the slit, at a position angle of 111.48 east of north to avoid possible photon contamination from a neighboring bright object $2^{\prime \prime}$ away. An A0V calibration star (HD 215143) was observed immediately after the exposures of HQT-9. The data were reduced using the REDSPEC package developed by the NIRSPEC instrument group at the UCLA IR lab. ${ }^{11}$ The resulting spectrum had poor

\footnotetext{
10 http://www.naoj.org/Observing/Instruments/OHS/spec/skyspec.html

11 REDSPEC is available at

http://www2.keck.hawaii.edu/inst/nirspec/redspec.html.
} 


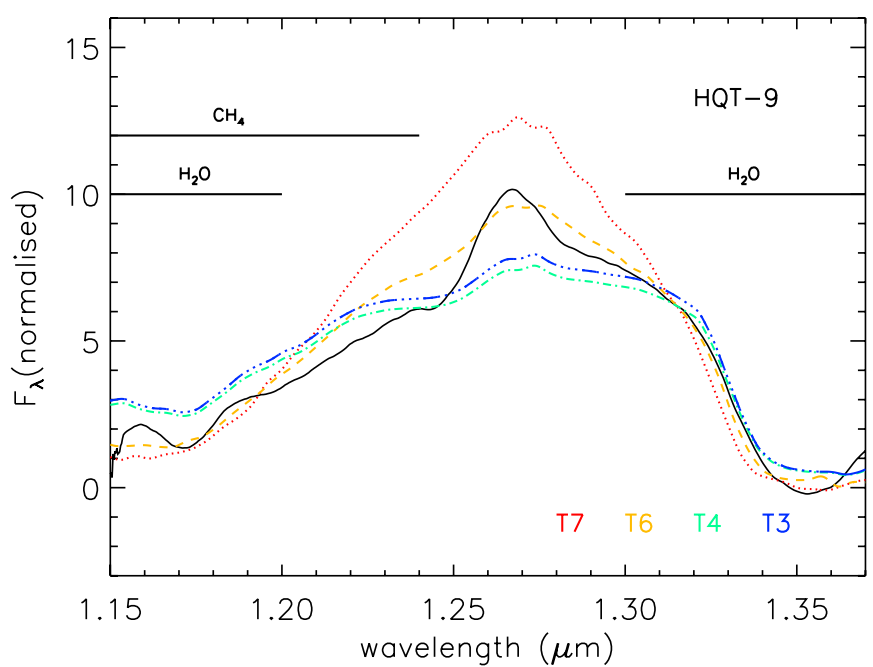

Figure 7. Keck/NIRSPEC $J$-band spectrum of HQT-9 (black solid curve). This object appears to be T4-T6, based on comparison with the dwarf standard spectra of Burgasser et al. (2006) (T7 [2MASS J07271824+1710012]: red dotted curve, T6 [SDSS J162414.37+002915.6]: orange dashed curve, T4 [2MASS 22541892+3123498]: green dash-dotted curve, T3 [2MASS J12095613-1004008]: blue dash-dot-dot-dotted curve).

(A color version of this figure is available in the online journal.)

$\mathrm{S} / \mathrm{N}$ and was subsequently smoothed with a boxcar of 50 pixels, yielding a spectral resolution of $R \approx 100$ for the final spectrum. Figure 7 shows the final spectrum of HQT-9.

\section{ANALYSIS}

\subsection{Classification with Colors}

The $z-J$ color information is essential to separate candidate quasars from candidate $\mathrm{T}$ dwarfs. Furthermore, in the case of $\mathrm{L}$ and $\mathrm{T}$ dwarfs, $z-J$ provides a better constraint on spectral types than $I-z^{\prime}$. This is because the photometric errors in optical bandpasses are generally larger than those in near-IR bandpasses for these ultracool dwarfs which emit the majority of their light in the near-IR. We thus obtained follow-up $J$-band imaging data for all of our candidates. Figure 8 presents their $I-z^{\prime}$ versus $z^{\prime}-J$ color-color diagram. The symbols used follow those of Figure 2. The shaded area denotes the expected color range for $\mathrm{T}$ dwarfs, and it is now well separated from the model high- $z$ quasar track. $H$-band imaging data exist for six candidates, and they are shown as red symbols in the $z^{\prime}-J$ versus $J-H$ diagram (Figure 9). Similarly, the $z^{\prime}-J$ versus $J-K$ color plot is shown in Figure 10.

Using these color-color diagrams, we classified objects into three categories: QSOs, L-type dwarfs, and T-type dwarfs. In the case of the L- and T-type dwarfs, we further estimated their spectral types using a color-spectral-type relation obtained from known dwarfs. This relation is shown as the pink dashed line in Figures 2, 8, and 10, and was derived by fitting colors of known dwarfs as functions of their spectral types with seventh-order polynomials. Optical colors of known dwarfs were computed from the spectra as our filter sets $\left(I, N B 816, z^{\prime}\right)$ are unique, and the procedure is described in Section 2. Table 1 lists the coefficients of the polynomial fits to colors. Near-IR colors come from photometric measurements of known $\mathrm{L}$ and $\mathrm{T}$ dwarfs from Knapp et al. (2004). We used the AB magnitude system for optical bandpasses of the Suprime-Cam $\left(I, N B 816, z^{\prime}\right)$, and the MKO [Vega] system for the MKO near-IR filter set $(J, H, K)$.

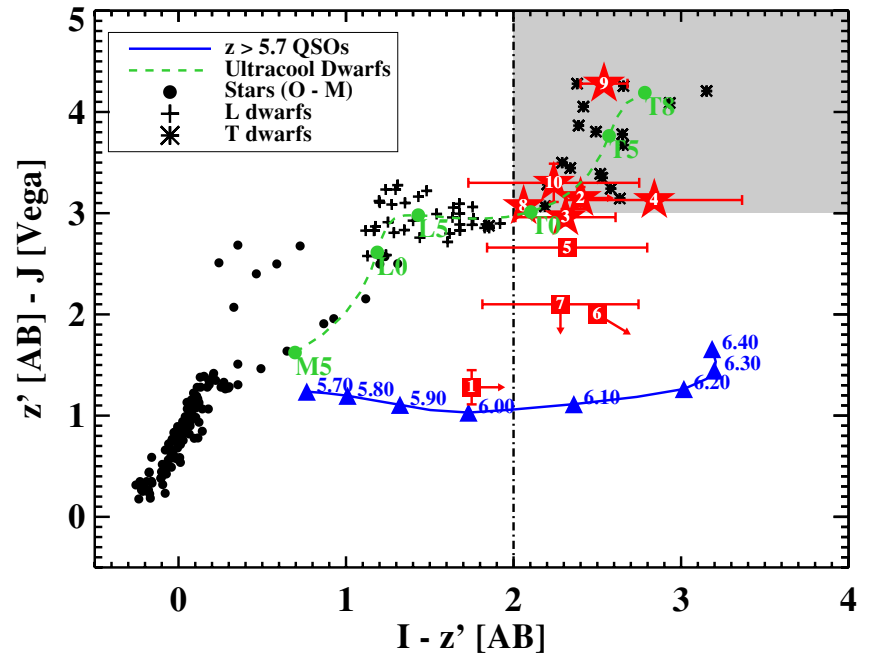

Figure 8. $I-z^{\prime}$ vs. $z^{\prime}-J$ color-color diagram for our candidates. Symbols are the same as in Figure 2, but in this color representation the shaded region only contains T dwarfs. Although a narrowband NB816 filter helps to separate $\mathrm{M} / \mathrm{L}$ dwarfs from high- $z$ quasar candidates, there is still a color degeneracy between quasars and T dwarfs (see Figure 2). This plot demonstrates that the $z^{\prime}-J$ color can cleanly break this degeneracy.

(A color version of this figure is available in the online journal.)

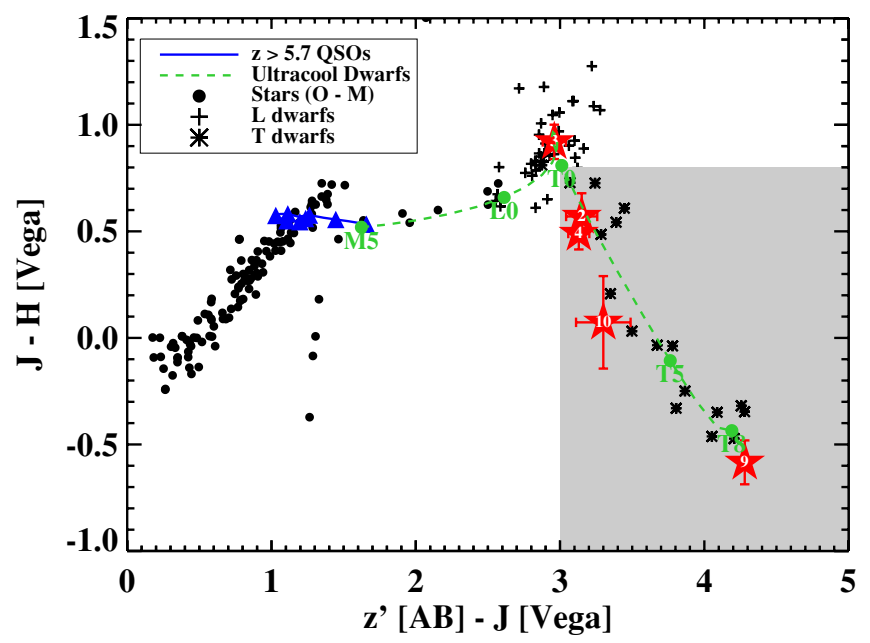

Figure 9. $z^{\prime}-J$ vs. $J-H$ diagram shown with the same symbols as in Figure 2. Five HQT candidates with $H$-band imaging data are plotted with numbered stars and error bars, and they lie in the shaded region of ultracool dwarfs. Note that errors are much smaller than in the optical. This is because ultracool dwarfs are much brighter in the near-IR wavelengths. The colors of $z>5.7$ quasars are degenerated with $\mathrm{M}$ stars. T dwarfs are characterized by their red $z^{\prime}-J$ color. However in $J-H$ color, they become increasingly blue at later spectral types.

(A color version of this figure is available in the online journal.)

The candidate classification based on their color information is shown in the "Class" section of Table 5. Six candidates out of a total of 10 are probable T dwarfs, though one candidate (HQT3 ) could be a late-type $\mathrm{L}$. These probable $\mathrm{T}$ dwarfs are shown with star symbols in Figures 2, 8, and 10, and are marked with asterisks in Tables 3 and 5. The remaining three candidates are tentatively classified as high- $z$ quasars, though optical spectra are necessary to confirm if they are truly quasars. One quasar candidate, HQT-6, could be a transient such as a Kuiper Belt object or supernova. This is because the $z^{\prime}$-band images of this object were not taken during the same observing run as its $I$ and NB816-band images, and HQT-6 was only detected in $z^{\prime}$. 
Table 5

Classification and Distance Measurements

\begin{tabular}{|c|c|c|c|c|c|c|}
\hline \multirow[t]{2}{*}{ Name } & \multirow[t]{2}{*}{ Class } & \multirow[t]{2}{*}{ Sp Type } & \multirow[t]{2}{*}{ Adopted Type } & \multicolumn{3}{|c|}{ Distance (pc) } \\
\hline & & & & L06-bright & L06-faint & L10 \\
\hline HQT-1 & QSO & $\ldots$ & $\ldots$ & $\ldots$ & $\ldots$ & $\ldots$ \\
\hline HQT-2* & T0-T3 & $\mathrm{T} 2-\mathrm{T} 4$ & $\mathrm{~T} 2-\mathrm{T} 4$ & $157 \pm 48.6$ & $115 \pm 30.3$ & $134 \pm 37.8$ \\
\hline HQT-3* & L7 -T0 & L9-T0 & L9-T0 & $119 \pm 29.5$ & $96.6 \pm 20.4$ & $109 \pm 24.8$ \\
\hline HQT-4* & $\mathrm{T} 1-\mathrm{T} 3$ & $\ldots$ & $\mathrm{T} 1-\mathrm{T} 3$ & $133 \pm 40.6$ & $96.5 \pm 25.0$ & $114 \pm 31.6$ \\
\hline HQT-5 & Late L & $\ldots$ & L7-L9 & $138 \pm 47.2$ & $127 \pm 40.8$ & $138 \pm 43.0$ \\
\hline HQT-6 & Transient or QSO & $\ldots$ & $\ldots$ & $\ldots$ & $\ldots$ & $\ldots$ \\
\hline HQT-7 & QSO & $\ldots$ & $\ldots$ & $\ldots$ & $\ldots$ & $\ldots$ \\
\hline HQT-8* & $\mathrm{T} 0-\mathrm{T} 2$ & $\ldots$ & T0-T2 & $131.0 \pm 45.3$ & $101 \pm 32.9$ & $115 \pm 36.6$ \\
\hline HQT-9* & T4-T9 & T4-T6 & T4-T6 & $104 \pm 33.4$ & $86.3 \pm 23.6$ & $93.5 \pm 27.3$ \\
\hline HQT-10* & T3-T5 & $\ldots$ & T3-T5 & $160 \pm 53.3$ & $123 \pm 35.6$ & $139 \pm 42.7$ \\
\hline
\end{tabular}

Notes. The "Class" was determined from the optical and near-IR colors of the objects, whereas the "Sp type" was decided based on the near-IR spectra. We gave precedence to "Sp type" for the candidates with near-IR spectra. Final spectral types for candidate brown dwarfs are listed in "Adopted Type." Distances are measured using relationships between absolute magnitude and spectral types from Liu et al. (2006) (L06-A and L06-B) and M. C. Liu et al. (in preparation, L09).

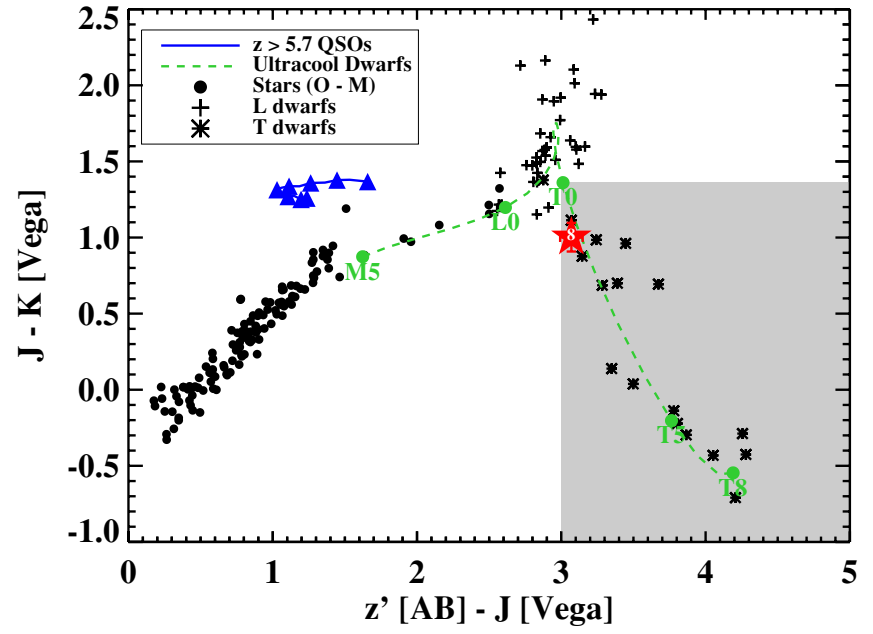

Figure 10. $z^{\prime}-J$ vs. $J-K$ diagram shown with the same symbols as in Figure 2. Only one candidate in Table 3 (HQT 8) was observed in the $K$ band and is plotted in this diagram. A similar color trend for $J-K$ colors with $z^{\prime}-J$ is seen for ultracool dwarfs that are plotted in $J-H$ in Figure 9, although typical $J-K$ color values are about half a magnitude larger at the same $z^{\prime}-J$ color. This is because the increasing gravity/metallicity in the later $\mathrm{T}$ dwarfs suppresses the $K$-band flux.

(A color version of this figure is available in the online journal.)

The Keck/DEIMOS spectra were obtained for two candidate quasars (HQT-1 and HQT-7), and they will be discussed in a forthcoming paper (Y. Kakazu et al., in preparation). This paper focuses on the ultracool dwarfs found by the HQT survey.

\subsection{Classification of Ultracool Dwarfs with Near-IR Spectra}

In order to confirm that our $\mathrm{T}$ dwarf candidates are truly ultracool objects, we have obtained near-IR spectra for three probable T dwarfs, HQT-2, HQT-3, and HQT-9. HQT-2 and HQT-3 were observed with Subaru/CISCO with the $J H$ grism and their spectra are presented in Figures 5 and 6 . Both objects clearly exhibit $\mathrm{CH}_{4}$ and $\mathrm{H}_{2} \mathrm{O}$ absorption, which are the hallmark features of T dwarfs. Since the S/N of our spectra is low, we classify the objects by directly comparing their overall spectral shapes with those of standard template $\mathrm{T}$ dwarf spectra from Burgasser et al. (2006), instead of using spectral diagnostic indices. The closest fit to HQT-2 appears to be T3, though there is a \pm 1 subclass uncertainty in the spectral type due to poor $S / N$ in the spectra. Similarly, we assign a spectral type of L9-T0 to HQT-3.

Figure 7 shows the Keck/NIRSPEC J-band spectrum of HQT9 with standard T dwarf spectra from Burgasser et al. (2006) overlaid. The spectra were obtained under non-photometric, strong wind conditions, which resulted in poor $\mathrm{S} / \mathrm{N}$. However, the detection of $\mathrm{CH}_{4}$ and $\mathrm{H}_{2} \mathrm{O}$ absorption bands ensures that this is a T dwarf. The closest template spectra is T6. The absorption feature below $1.26 \mu \mathrm{m}$ is likely an artifact of low $\mathrm{S} / \mathrm{N}$ data. We therefore assign a broad spectral type of T4-T6 to HQT-9. All three T dwarfs that are spectroscopically confirmed have spectral types consistent with those derived from their optical/near-IR colors. Table 5 lists spectral classifications for the $\mathrm{L} / \mathrm{T}$ candidates derived from colors and spectra.

\subsection{Distance Measurements}

In general, distance estimates of field brown dwarfs with no parallax measurements are made using the absolute magnitude versus spectral-type relations obtained from nearby brown dwarfs (Dahn et al. 2002; Tinney et al. 2003; Vrba et al. 2004). It has been observationally known that the dwarfs between latetype $\mathrm{L}$ and early-type $\mathrm{T}$ show an unusual strong brightening $(\sim 1 \mathrm{mag})$ in their $J$-band absolute magnitudes. A similar brightening appears in other wavelengths $\left(z^{\prime}\right.$ and $H$ ), though the effect is the greatest in the $J$ band. The identifications of $\mathrm{L} / \mathrm{T}$ transition binaries by Liu et al. (2006) and Burgasser et al. (2007) suggest that this brightening may be enhanced by a high incidence of unresolved binaries at $\mathrm{L} / \mathrm{T}$ transition and that the true brightening amplitude is more modest. A growing number of resolved binaries support this claim (M. C. Liu et al. 2010, in preparation). Here, to estimate the distances of the HQT candidates, we use the absolute magnitude versus spectral-type relations from Liu et al. (2006) and M. C. Liu et al. (2010, in preparation). Liu et al. (2006) present two sets of polynomial fits-one derived after removing known binaries in the Knapp et al. (2004) sample (L06-bright) and another derived after removing known and possible binaries (L06-faint). The latter fits show almost no brightening "hump" at L/T transitions, and thus the distances derived from these fits are smaller than those derived from the former fits. The new relations of M. C. Liu et al. (2010, in preparation, L10) are closer to L06-faint relations, however, have slightly larger brightening amplitudes. 
Table 6

Proper Motions and Tangential Velocities

\begin{tabular}{lccccc}
\hline \hline Name & & & \multicolumn{3}{c}{$v_{\tan }$} \\
\cline { 4 - 6 } & $\begin{array}{c}\Delta T \\
(\mathrm{yr})\end{array}$ & $\begin{array}{c}\mu \\
\left(\mathrm{mas} \mathrm{yr}^{-1}\right)\end{array}$ & $\begin{array}{c}\text { L06-bright } \\
\left(\mathrm{km} \mathrm{s}^{-1}\right)\end{array}$ & $\begin{array}{c}\text { L06-faint } \\
\left(\mathrm{km} \mathrm{s}^{-1}\right)\end{array}$ & $\begin{array}{c}\text { L10 } \\
\left(\mathrm{km} \mathrm{s}^{-1}\right)\end{array}$ \\
\hline HQT-1 & 2.9 & $\ldots$ & $\ldots$ & $\ldots$ & $\ldots$ \\
HQT-2 & 1.1 & $130 \pm 97$ & $96.8 \pm 78.2$ & $70.9 \pm 56.1$ & $82.6 \pm 65.9$ \\
HQT-3 & $\ldots$ & $\ldots$ & $\ldots$ & $\ldots$ & $\ldots$ \\
HQT-4 & 3.0 & $47 \pm 25$ & $29.7 \pm 18.2$ & $21.5 \pm 12.7$ & $25.4 \pm 15.2$ \\
HQT-5 & 1.9 & $77 \pm 33$ & $50.5 \pm 27.7$ & $46.2 \pm 24.8$ & $50.2 \pm 26.7$ \\
HQT-6 & $\ldots$ & $\ldots$ & $\ldots$ & $\ldots$ & $\ldots$ \\
HQT-7 & $\ldots$ & $\ldots$ & $\ldots$ & $\ldots$ & $\ldots$ \\
HQT-8 & 2.2 & $44 \pm 21$ & $27.8 \pm 15.8$ & $20.5 \pm 11.1$ & $24.0 \pm 13.8$ \\
HQT-9 & 3.6 & $170 \pm 10$ & $83.6 \pm 28.5$ & $69.5 \pm 20.5$ & $75.3 \pm 23.6$ \\
HQT-10 & 1.7 & $66 \pm 28$ & $50.0 \pm 27.0$ & $38.5 \pm 19.8$ & $43.5 \pm 22.8$ \\
\hline
\end{tabular}

Notes. $\Delta T$ means observation time baseline. Tangential velocities were estimated using the distances presented in Table 5.

The brightening effect is more modest in the $H$-band than in the $J$-band, and thus the uncertainty caused by an unknown binary fraction is smaller. Hence we used apparent $H$-band magnitudes to estimate the distances for the HQT candidates with $H$-band photometry. For objects without $H$-band data (HQT-5 and HQT-8), $J$-band photometry was used. Table 5 shows the resulting distance measurements based on the three relations described above (L06-bright, L06-faint, and L10). The errors in distance measurements are calculated as a combination of the $J$-band photometric errors, the errors due to spectral type uncertainties in classifications, and the $M_{H}$ (or $M_{J}$ ) scatter in the absolute magnitude versus spectral type relations. Three spectroscopically confirmed T dwarfs (HQT-2, HQT-3, HQT9) have distances of $60-170 \mathrm{pc}$, though HQT-3 could be a late-type $\mathrm{L}$.

Of 200 known T dwarfs, there are only a handful of spectroscopically confirmed $\mathrm{T}$ dwarfs known today at distances beyond 60 pc: ULASJ1452+0655 (T4.5) at $83 \mathrm{pc}$ (Kendall et al. 2007), SOri 70 (T6) at $400 \mathrm{pc}$ (Zapatero Osorio et al. 2002) - though this was later disputed by Burgasser et al. (2004) to be at 75-100 pc-and NTTDF1205-0744 (T6) at $90 \mathrm{pc}$ (Cuby et al. 1999). In addition are, two T dwarfs from UKIDDS DR1 at around $60 \mathrm{pc}$ (ULAS J100759.90-010031.1 [T5.5] and ULAS J223955.76+003252.6 [T4.5]; Lodieu et al. 2007), and recently a dozen $\mathrm{T}$ dwarfs from UKIDSS DR4 (Burningham et al. 2010). Three spectroscopically confirmed $T$ dwarfs found by our survey are among these most distant, spectroscopically confirmed $\mathrm{T}$ dwarfs. The discovery of distant $\mathrm{T}$ dwarfs from the HQT survey demonstrates the effectiveness of our optical color selection.

\subsection{Proper Motion}

Since there is a greater than one year epoch difference between the Suprime-Cam optical imaging survey and the $J$-band follow-up observations, it is possible to place limits on proper motions and hence tangential velocities for our candidates. Space velocities of stars and substellar objects are expected to increase as they age through interaction with Galactic disk stars. Therefore, proper motion measurements combined with photometric distance estimates of field ultracool dwarfs can provide insight into their age and distribution in the Galaxy. For instance, at a given spectral type, a lower velocity suggests a younger, lower-mass object, while a higher velocity suggests an older, higher-mass object. The detection of a high proper motion ultracool dwarf might imply that it is an old member of the Galactic disk population (Burgasser et al. 2007). An extremely high proper motion might indicate membership in a low-metallicity, halo population. Furthermore, proper motion measurements can be used as a way of separating out ultracool dwarfs from high- $z$ galaxies/quasars that have similar optical colors as dwarfs, since extragalactic objects have zero proper motions.

In order to measure proper motion, we need to register a follow-up $J$-band image of each candidate onto its SuprimeCam $z^{\prime}$-band image. The registration can be done using the field stars which appear in both $J$ - and $z^{\prime}$-band images. We chose such astrometric reference stars in the following way. We first selected objects that lie within $2^{\prime}$ of each candidate. This area constraint was set to minimize the effect of non-linear distortion. We then choose objects that are detected in both the optical $z^{\prime}$ and the $J$ band at $>10 \sigma$, but are not saturated. We finally selected objects whose SExtractor star parameters are greater than 0.8 and with flags parameters of zero. These criteria yielded typically 15-20 astrometric reference stars for each candidate.

Using these reference stars, we derived the third-order transformation coefficients between the $J$-band image and the $z^{\prime}$ band image. ${ }^{12}$ The astrometric registration tool in the SIMPLE software was used in this process, which finds a solution in an iterative manner by rejecting stars with registration errors greater than $3 \sigma$. Proper motion was then measured by directly comparing absolute coordinates of each candidate in the Suprime-Cam $z^{\prime}$ and in the registered $J$ images. The errors in proper motion were computed by taking a median of position differences of reference stars between $z^{\prime}$ and $J$ images. We also measured proper motion independently by measuring the change in the median of distances between a candidate and its astrometric reference stars. This alternative measurement was carried out in order to examine a possible systematic offset between $J$ - and $z^{\prime}$-band images arising from the registration. However, this method yielded consistent results with the previous method within $1 \sigma$ errors. In the following discussion, we adopt the values derived by the direct comparison of candidate absolute coordinates in the $z^{\prime}$ and $J$-band images.

Table 6 summarizes the results of our proper motion measurements in units of milliarcsecond (mas) per year. Note that none of the candidate quasars were examined for motions since their $J$-band magnitudes are too faint $(\leqslant 5 \sigma)$ to unambiguously measure their positions in the follow-up $J$-band images. The proper motion of HQT-3 was not measured since its $J$-band image lacks a sufficient number of reference stars to register onto the $z^{\prime}$-band image. $\mathrm{L}$ and $\mathrm{T}$ dwarfs found in our survey are among the most distant known ultracool dwarfs, and hence a long time baseline $\Delta T$ is required to accurately measure their proper motion. As such, the short baseline $\Delta T$ of our observations have resulted in large errors in proper motions, and consequently in tangential velocities, $V_{\tan }=4.74 \mu d \mathrm{~km} \mathrm{~s}^{-1}$. Future follow-up observations are necessary to minimize the errors and to draw reliable conclusions. However, the spectroscopically confirmed T dwarf with the longest baseline observations (HQT-9; T4-T6; $\Delta T=3.6 \mathrm{yr}$ ) in our sample, has a reasonably good proper

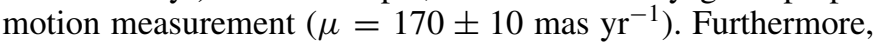

\footnotetext{
12 Note that the third-order polynomial transformation function used in the SIMPLE software is defined as $\sum_{i, j} x^{i} \times y^{i}$, where $(x, y)$ is a coordinate, and $i+j \leqslant 3$. This is different from some other forms of third-order polynomial fits, where $i \leqslant 3$ and $j \leqslant 3$. In the former transform, there are 20 free parameters (coefficients), while the latter requires twice as many coefficients to be constrained.
} 

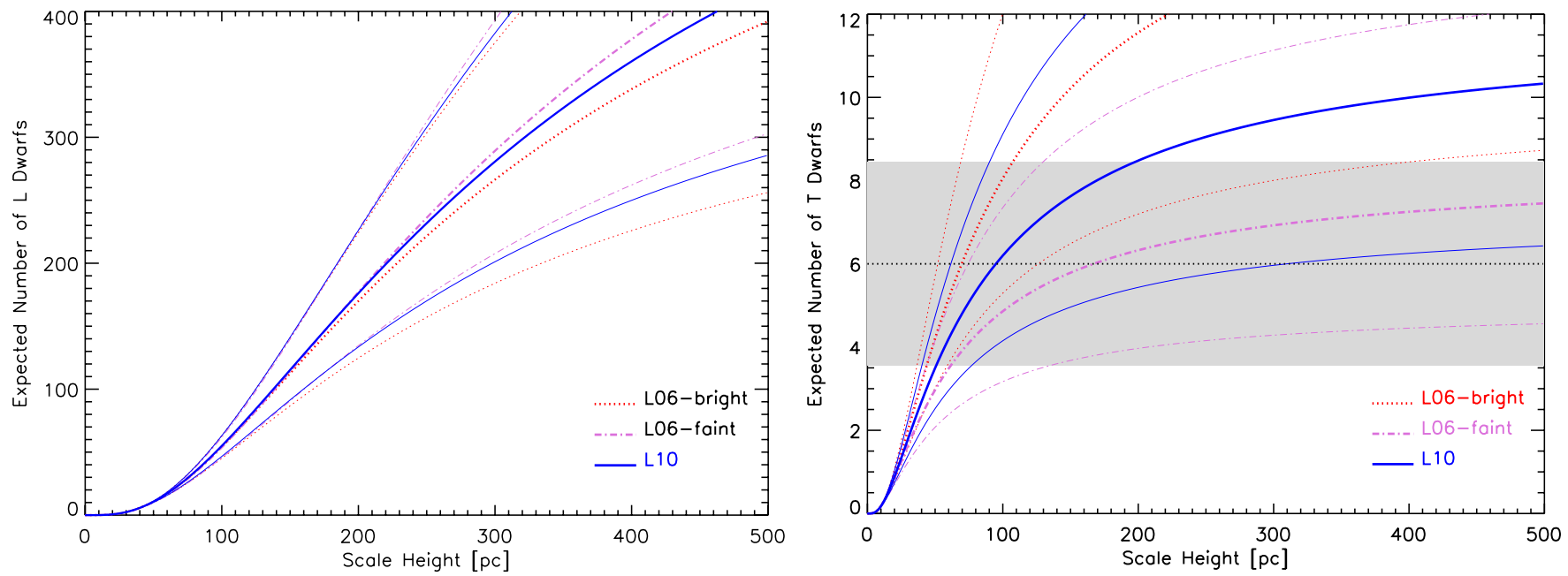

Figure 11. Expected number of L dwarfs (left) and T dwarfs (right) as a function of Galactic scale height. Three kinds of $M_{K}$ vs. spectral-type relations from Liu et al. (2006) (L06-bright and L06-faint) and M. C. Liu et al. (2010, in preparation, L10) are used for distance estimates. The expected number of dwarfs from each relation is shown as a red dotted curve (L06-bright), pink dash-dotted curve (L06-faint), and blue solid curve (L10). Thin lines represent the $1 \sigma$ uncertainties. It can be seen that the differences in these relations result in a large difference in expected numbers of T dwarfs (right), but not in L dwarfs (left). Our findings of six T dwarfs with $1 \sigma$ Poisson error ranges are shown by the shaded region in the right-hand plot.

(A color version of this figure is available in the online journal.)

this object was observed at three different epochs (SuprimeCam; UT 2003.10.26 and UT 2005.09.05 and MOIRCS; UT 2007.06.12), allowing three independent proper motion measurements, which all agree with each other within the errors. Here, we adopt the proper motion derived from a linear fit to the available three epochs.

The tangential velocity of HQT-9 $\left(V_{\tan }=65 \pm 14 \mathrm{~km} \mathrm{~s}^{-1}\right)$ is more than two times larger than the median value of nearby $(<20 \mathrm{pc}) \mathrm{T}$ dwarfs in Faherty et al. (2009), suggesting it is older and more massive than the typical $\mathrm{T}$ dwarfs. The remaining candidate $\mathrm{L}$ and $\mathrm{T}$ dwarfs appear to have velocities consistent with Faherty et al. (2009; $V_{\tan }=20-60 \mathrm{~km} \mathrm{~s}^{-1}$ for T dwarfs), though given the large errors, future follow-up observations are necessary. The large velocity of the newly found T dwarf, HQT9 , and the lack of T dwarfs with small velocities may imply that $\mathrm{T}$ dwarfs are old members of the Galactic disk.

\subsection{Number Counts and Galactic Scale Height of Cool Dwarfs}

Few studies have been conducted so far on the vertical scale height of $\mathrm{L}$ and $\mathrm{T}$ dwarfs, as this requires probing distant dwarfs over wide areas at different Galactic latitudes (e.g., Liu et al. 2002). Ryan et al. (2005) estimated the scale height to be $350 \pm 50 \mathrm{pc}$ based on 28 faint $\mathrm{L}$ and $\mathrm{T}$ dwarf candidates $\left(21<z^{\prime}<25\right)$ found in the deep Hubble Space Telescope/ Advanced Camera for Surveys parallel fields. Their estimate is consistent with the known trend of increasing scale height with decreasing stellar mass. However, given the small area coverage $\left(\sim 0.04 \mathrm{deg}^{2}\right)$, ideally one would like to have dwarfs from a wider area.

We have identified six T dwarfs from the HQT survey, of which three were spectroscopically confirmed including one possibly late L dwarf, HQT-3. Although the number of detected ultracool dwarfs by the HQT survey is still small, our survey area coverage of $\sim 10 \mathrm{deg}^{2}$ is significantly larger than the previous work on the vertical scale height $\left(\sim 0.5 \mathrm{deg}^{2}\right.$ of IfA deep survey in Liu et al. 2002; 10 $\operatorname{arcmin}^{2}$ of HUDF in Pirzkal et al. 2005; $\sim 0.04 \mathrm{deg}^{2}$ in Ryan et al. 2005). Thus, our data may provide a better constraint on the scale height. Using a simple analytic model of the Galactic disk, we estimate the expected number of dwarfs and examine if the HQT survey can set a useful limit on the Galactic scale height of these ultracool dwarfs.

Following the steps described in Liu et al. (2002), we calculate the expected number of L and T dwarfs from the HQT survey as a function of Galactic scale height. We first assume that the distribution of $\mathrm{T}$ dwarfs follows the exponential Galactic disk model of Wainscoat et al. (1992) with a radial scale length of $3.5 \mathrm{kpc}$. We can estimate the maximum distances of dwarfs that can be probed by the HQT survey from the survey magnitude limit of $z^{\prime}=23.3$ and the absolute magnitude versus spectraltype relations. The effect of brightening at $\mathrm{L} / \mathrm{T}$ transitions is more modest in the $K$ band than that in the $J$ band. As such, the uncertainty in the brightening amplitude due to unrecognized binaries is expected to be smaller in the $K$ band. Hence, we use the $M_{K}$ versus spectral-type relations for our analysis and transform $M_{K}$ to $M_{z^{\prime}}$ using the $z^{\prime}-K$ synthesized colors of dwarfs presented in Table 1 .

With the L10 relation, we can probe L dwarfs out to $700 \mathrm{pc}$ and T dwarfs out to $190 \mathrm{pc}$. If we use the L06-bright relations, the probable distances become slightly larger ( $\mathrm{T}$ dwarfs out to $200 \mathrm{pc}$ ) due to a larger brightening amplitude in the early $\mathrm{T}$ dwarfs. Consequently the survey volume becomes larger for the L06-bright relations. As a result, the number of dwarfs found by the survey is expected to be larger with the L06-bright relations. We carry out our number counts analysis based on three relations (L06-bright, L06-faint, and L10). We adopt local spatial density of $\mathrm{L}$ and $\mathrm{T}$ dwarfs tabulated by Caballero et al. (2008).

Given these quantities, we now calculate the expected number of $\mathrm{T}$ dwarfs for each spectral subclass by multiplying the exponential disk density of Wainscoat et al. (1992) by the survey surface area and integrating it over the distance to which our survey is sensitive. Finally, we sum the expected number of each subclass of $\mathrm{T}$ dwarf to derive the number of the entire $\mathrm{T}$ dwarf population. Figure 11 presents the expected number of L dwarfs (left) and T dwarfs (right) in the entire HQT survey area as a function of the Galactic scale height of these dwarfs. Model predictions are presented as a red dotted line (L06- 

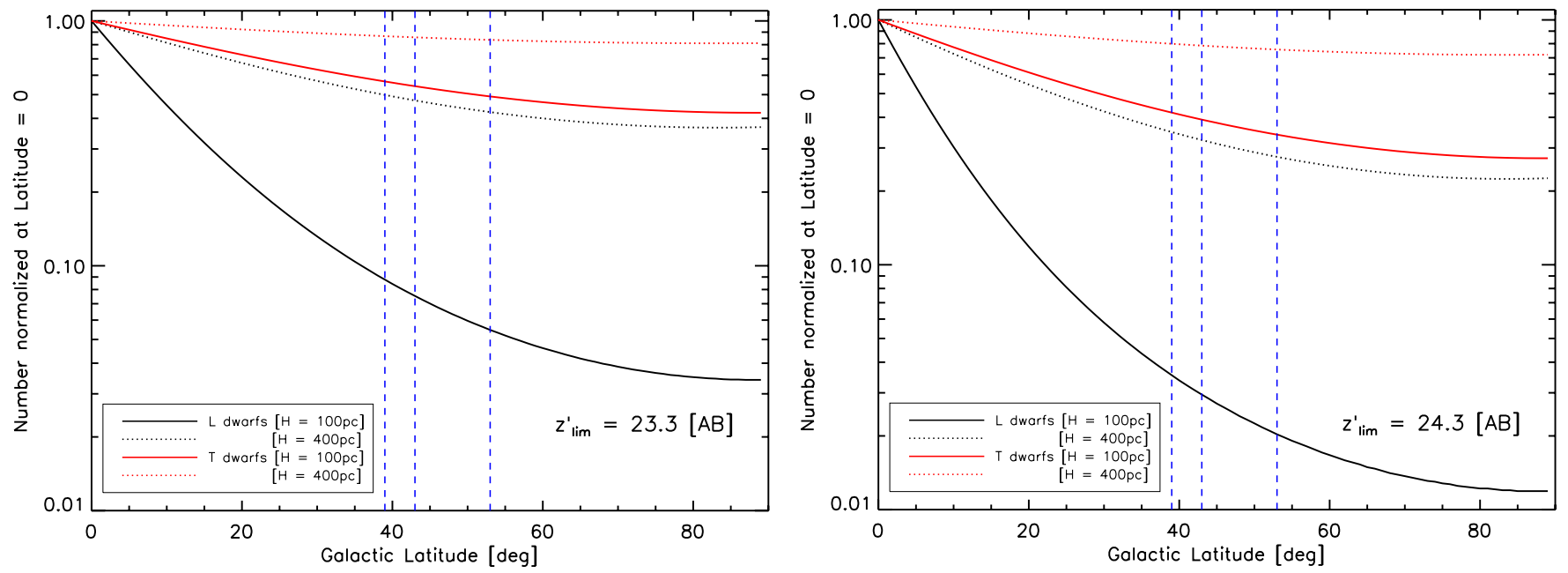

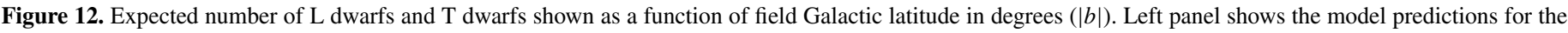


The locations of the three HQT survey fields are indicated with dashed blue lines.

(A color version of this figure is available in the online journal.)

bright), pink dash-dotted line (L06-faint), and blue solid line (L10). One sigma errors derived from the uncertainty in the absolute magnitude relations are also shown as thin lines. The effect of the use of different relations is almost negligible in the number of L dwarfs, but is clearly evident in that of T dwarfs. Our results suggest that the number of $\mathrm{L}$ dwarfs discovered by the HQT survey is sensitive to their Galactic scale height. However, this is not the case with $\mathrm{T}$ dwarfs where numbers become essentially constant at a scale height greater than $200 \mathrm{pc}$. This is because the HQT survey is only sensitive to $\mathrm{T}$ dwarfs within $200 \mathrm{pc}$.

Our findings of six T dwarfs in the HQT survey are indicated as a horizontal dotted line with a shaded area for the $1 \sigma$ Poisson statistical uncertainty. Although the large uncertainty is seen in the expected number of $\mathrm{T}$ dwarfs, our findings are consistent with model predictions.

It is known that the number ratio of dwarfs at different latitudes is a function of their Galactic scale height (Liu et al. 2002; Ryan et al. 2005). Thus by comparing the number of identified dwarfs in the fields at different latitudes, one may be able to set a useful limit on the scale height. We therefore examine the expected number of dwarfs at different Galactic latitudes. The purpose here is to understand how one's choice of survey field affects the survey sensitivity to the dwarfs' scale height. We follow the same procedure described above, except we now compute the expected number of $\mathrm{L}$ and $\mathrm{T}$ dwarfs as a function of both scale height and field latitude. The L10 relations are used to estimate the survey volume. The left panel of Figure 12 presents the results for our survey depths. In the right panel, we show the results for a survey one magnitude deeper to illustrate the effect of the survey depths. In both cases, the dwarf numbers are normalized at $0^{\circ}$ latitude and they are shown as black (L dwarfs) and red (T dwarfs) solid lines for the scale height of $100 \mathrm{pc}$. Dotted lines indicate the results for the scale height of $400 \mathrm{pc}$.

The three HQT survey fields are indicated with blue dotted lines. It can be seen from the left plot in Figure 12 that the $\mathrm{T}$ dwarf number ratio varies very little between the low- and high-latitude fields of the HQT survey for both scale heights. Thus, the HQT survey is not very sensitive to the scale height of T dwarfs. On the other hand, the $\mathrm{L}$ dwarf number ratio is a strong function of the scale height; the number ratio is almost 1 for a scale height of $400 \mathrm{pc}$, but it becomes 1.6 for a scale height of $100 \mathrm{pc}$.

This figure clearly demonstrates that by observing multiple fields at very different latitudes, one can set better constraints on the dwarf scale height. For example, number ratios of $\mathrm{L}$ dwarfs in a $|b|=10^{\circ}$ field and $|b|=80^{\circ}$ field are $\sim 13$ for a scale height of $100 \mathrm{pc}$. This becomes 2 for a scale height of $400 \mathrm{pc}$. In the case of $\mathrm{T}$ dwarfs, the number ratio changes from 2.5 to 1 . The right plot in Figure 12 shows the results for a survey one magnitude deeper than the HQT survey. Here, the number ratio becomes larger and therefore one will be able to set better constraints on the scale height. The T dwarf number ratio at low- $\left(|b|=10^{\circ}\right)$ and high-latitude $\left(|b|=80^{\circ}\right)$ fields is 4 if the scale height is $100 \mathrm{pc}$. This becomes 1 if the dwarf scale height is $400 \mathrm{pc}$.

\section{CONCLUSION}

We have shown that the addition of the NB816 filter to the traditional optical $\left(I, z^{\prime}\right)$ survey for high- $z$ quasars is beneficial for breaking a well-known color degeneracy between quasars and foreground $\mathrm{M}$ and $\mathrm{L}$ dwarfs. Our color selection is useful as it does not require follow-up $J$-band imaging which is still time consuming compared to wide-field optical mosaic CCD imaging. This paper discusses objects that were selected by their extremely red $I-z^{\prime}$ and $N B 816-z^{\prime}$ colors with the purpose of identifying $\mathrm{T}$ dwarfs and quasars at the highest redshift range probed by optical $z^{\prime}$-band surveys $(z>6.1)$. Among 10 such candidates, we have successfully identified 5 or 6 T dwarfs, of which 3 have been spectroscopically confirmed. These $\mathrm{T}$ dwarfs are among the most distant spectroscopically confirmed ultracool dwarfs. The proper motion measurements of these T dwarfs indicate that they are old members of Galactic disks, though future follow-up imaging is necessary given the large errors in our measurements.

We have examined the expected number of $\mathrm{L}$ and $\mathrm{T}$ dwarfs from the HQT survey using a simple analytic model in which dwarfs follow an exponential disk distribution. Our discovery rate is consistent with the model predictions, though the uncertainty is large due to small number statistics. We have also 
investigated the effect of survey field latitude on the determination of dwarf scale height. Our results show that multiple field surveys that cover a wide range of latitudes will be very useful in the determination of the scale height of dwarfs.

We are indebted to the staff of the Subaru, Keck, and UKIRT observatories for their excellent assistance with the observations. We especially thank the support astronomer, Hisanori Furusawa, for his invaluable help in completing the observing run with the Suprime-Cam. We are also grateful to the anonymous referee for a helpful report.

\section{REFERENCES}

Ajiki, M., et al. 2003, AJ, 126, 2091

Bertin, E., \& Arnouts, S. 1996, A\&AS, 117, 393

Burgasser, A. J., Geballe, T. R., Leggett, S. K., Kirkpatrick, J. D., \& Golimowski, D. A. 2006, ApJ, 637, 1067

Burgasser, A. J., Kirkpatrick, J. D., Liebert, J., \& Burrows, A. 2003, ApJ, 594, 510

Burgasser, A. J., Kirkpatrick, J. D., McGovern, M. R., McLean, I. S., Prato, L., \& Reid, I. N. 2004, ApJ, 604, 827

Burgasser, A. J., Looper, D. L., Kirkpatrick, J. D., \& Liu, M. C. 2007, ApJ, 658, 557

Burningham, B., et al. 2010, MNRAS, 406, 1885

Caballero, J. A., Burgasser, A. J., \& Klement, R. 2008, A\&A, 488, 181

Capak, P., et al. 2004, AJ, 127, 180

Casali, M., et al. 2007, A\&A, 467, 777

Chiu, K., Fan, X., Leggett, S. K., Golimowski, D. A., Zheng, W., Geballe, T. R., Schneider, D. P., \& Brinkmann, J. 2006, AJ, 131, 2722

Cuby, J. G., Saracco, P., Moorwood, A. F. M., D’Odorico, S., Lidman, C., Comerón, F., \& Spyromilio, J. 1999, A\&A, 349, L41

Cushing, M. C., Rayner, J. T., \& Vacca, W. D. 2005, ApJ, 623, 1115

Dahn, C. C., et al. 2002, AJ, 124, 1170

Delorme, P., et al. 2010, A\&A, 518, 39

Dye, S., et al. 2006, MNRAS, 372, 1227

Epchtein, N., et al. 1999, A\&A, 349, 236

Faherty, J. K., Burgasser, A. J., Cruz, K. L., Shara, M. M., Walter, F. M., \& Gelino, C. R. 2009, AJ, 137, 1

Fan, X., et al. 2001, AJ, 122, 2833

Fan, X., et al. 2003, AJ, 125, 1649

Fan, X., et al. 2006, AJ, 131, 1203

Geballe, T. R., et al. 2002, ApJ, 564, 466

Golimowski, D. A., et al. 2004, AJ, 127, 3516

Hu, E. M., Cowie, L. L., Capak, P., \& Kakazu, Y. 2005, in IAU Colloq. 199 Probing Galaxies through Quasar Absorption Lines, ed. P. Williams, C.-G. Shu, \& B. Menard (Cambridge: Cambridge Univ. Press), 363
Hu, E. M., Cowie, L. L., Capak, P., McMahon, R. G., Hayashino, T., \& Komiyama, Y. 2004, AJ, 127, 563

Hu, E. M., Cowie, L. L., Kakazu, Y., \& Barger, A. J. 2009, ApJ, 698, 2014

Hu, E. M., Cowie, L. L., McMahon, R. G., Capak, P., Iwamuro, F., Kneib, J., Maihara, T., \& Motohara, K. 2002, ApJ, 568, L75

Ichikawa, T., et al. 2006, Proc. SPIE, 6269

Kakazu, Y., Cowie, L. L., \& Hu, E. M. 2007, ApJ, 668, 853

Kendall, T. R., et al. 2007, A\&A, 466, 1059

Knapp, G. R., et al. 2004, AJ, 127, 3553

Ko, J., et al. 2009, ApJ, 695, L198

Lawrence, A., et al. 2007, MNRAS, 379, 1599

Leggett, S. K., et al. 2006, MNRAS, 373, 781

Liu, M. C., Leggett, S. K., Golimowski, D. A., Chiu, K., Fan, X., Geballe, T. R., Schneider, D. P., \& Brinkmann, J. 2006, ApJ, 647, 1393

Liu, M. C., Wainscoat, R., Martín, E. L., Barris, B., \& Tonry, J. 2002, ApJ, 568, L107

Lodieu, N., et al. 2007, MNRAS, 379, 1423

McLean, I. S., et al. 1998, Proc. SPIE, 3354, 566

Medezinski, E., Broadhurst, T., Umetsu, K., Oguri, M., Rephaeli, Y., \& Benítez, N. 2010, MNRAS, 405, 257

Miyazaki, S., et al. 2002, PASJ, 54, 833

Motohara, K., et al. 2002, PASJ, 54, 315

Oke, J. B. 1990, AJ, 99, 1621

Pickles, A. J. 1998, PASP, 110, 863

Pinfield, D. J., et al. 2008, MNRAS, 390, 304

Pirzkal, N., et al. 2005, ApJ, 622, 319

Puget, P., et al. 2004, Proc. SPIE, 5492, 978

Ramsay Howat, S. K., et al. 2004, Proc. SPIE, 5492, 1160

Ryan, R. E., Jr., Hathi, N. P., Cohen, S. H., \& Windhorst, R. A. 2005, ApJ, 631, L159

Sharp, R. G., Crampton, D., Hook, I. M., \& McMahon, R. G. 2004, MNRAS, 350,449

Skrutskie, M. F., et al. 1997, in The Impact of Large Scale Near-IR Sky Surveys, ed. F. Garzon et al. (Astrophysics and Space Science Library, Vol. 210; Dordrecht: Kluwer), 25

Songaila, A., \& Cowie, L. L. 2002, AJ, 123, 2183

Tinney, C. G., Burgasser, A. J., \& Kirkpatrick, J. D. 2003, AJ, 126, 975

Tokunaga, A. T., \& Vacca, W. D. 2005, PASP, 117, 421

Vanden Berk, D. E., et al. 2001, AJ, 122, 549

Vrba, F. J., et al. 2004, AJ, 127, 2948

Wainscoat, R. J., Cohen, M., Volk, K., Walker, H. J., \& Schwartz, D. E. 1992, ApJS, 83, 111

Wang, W., Cowie, L. L., Barger, A. J., Keenan, R. C., \& Ting, H. 2010, ApJS, 187,251

Willott, C. J., Delfosse, X., Forveille, T., Delorme, P., \& Gwyn, S. D. J. 2005, ApJ, 633, 630

York, D. G., et al. 2000, AJ, 120, 1579

Zapatero Osorio, M. R., Béjar, V. J. S., Martín, E. L., Rebolo, R., Barrado y Navascués, D., Mundt, R., Eislöffel, J., \& Caballero, J. A. 2002, ApJ, 578, 536 